\title{
A Hub Signaling Pathway of Antimicrobial- Antifungal-Anticancer Peptides Axis With Cationic Residue Amino Acids on N, C-Terminals Under 500 Dalton Rule Via Network Pharmacology
}

Ki Kwang Oh

Kangwon National University

Md. Adnan

Kangwon National University

Dong Ha Cho ( $\square$ chodh@kangwon.ac.kr)

Kangwon National University

\section{Research Article}

Keywords: Short cationic peptides, Antimicrobial peptides-Antifungal peptides-Anticancer peptides axis, HPIK, STAT3, HVTK, NOS2, HIF-1 signaling pathway

Posted Date: June 17th, 2021

DOl: https://doi.org/10.21203/rs.3.rs-620485/v1

License: (c) (1) This work is licensed under a Creative Commons Attribution 4.0 International License.

Read Full License 


\section{Abstract}

Background: Short cationic peptides (SCPs) with therapeutic efficacy of Antimicrobial peptides (AMPs), Antifungal peptides (AFPs), and Anticancer peptides (ACPs) are known as enhancement of host defense system. Here, we investigated the uppermost peptide(s), hub signaling pathway(s), and its associated target(s) through network pharmacology.

Method: Firstly, we selected SCPs with positive amino acid residues on N-, C- terminals under 500 Dalton via RStudio. Secondly, EMBOSS pepstats, PASTA 2.0 and Aggrescan were used to remove non- AMPs, after that, ADAM, dbAMP, DBAASP v3.0, and MLAMP were utilized for AMPs selection. AMPs-targets were identified from both SEA and STP databases. The overlapping targets between the bacteria-responsive targets (TTD and OMIM) and AMPs-targets were visualized by VENNY 2.1. Thirdly, AFPs were filtered through Antifp tool, and TTD and OMIM selected fungal responsive targets. The overlapping targets between AFPs-targets and fungal-responsive targets were visualized by VENNY 2.1. Fourthly, the overlapping targets between cancer-related targets (TTD and OMIM) and fungal-responsive targets were visualized by VENNY 2.1. Fifthly, signaling pathway analysis of overlapping targets was performed via RStudio. Finally, molecular docking study (MDS) was carried out to discover the most potent peptides on a hub signaling pathway.

Results: A total of 1,833 SCPs were identified, and AMPs, AFPs, and ACPs filtration suggested that 197 peptides-30 targets, 81 peptides- 6 targets, and 59 peptides-4 targets are connected, respectively. The AMPs-AFPs-ACPs axis indicated that 27 peptides-2 targets are associated. Each hub signaling pathway for enhancement of host defense system was "Inactivation of Rap1 signaling pathway on AMPs", "Activation of Notch signaling pathway on AMPs-AFPs axis", and "Inactivation of HIF-1 signaling pathway on AMPs-AFPs-ACPs axis". The most potent peptides were assessed via MDS; finally, HPIK on STAT3, HVTK on NOS2 manifested the HIF-1 signaling pathway's highest affinity. Furthermore, the two peptides have better affinity scores than standard selective inhibitors (Stattic, 1400W).

Conclusion: Overall, the most potent SCPs for the host defense system were HPIK on STAT3 and HVTK on NOS2, which might inactivate the HIF-1 signaling pathway.

\section{Introduction}

Since the emergence of insulin application in the 1920s, peptide therapeutics have been revealed as highly selective, safe, efficacious, and well-tolerated pharmaceutical agents ${ }^{1}$. Peptides are intrinsic signaling molecules, possess both biochemical and therapeutical attribution, and nearly more than 60 peptides are being used (FDA approved) worldwide as a clinical medication ${ }^{2}$. Peptides' critical properties as potential drug candidates are their high potency on target disease, specificity on a target protein, and minimal toxicity ${ }^{3}$. Certainly, peptides provide potential therapeutic intervention by binding to particular cell surface receptors which stimulate intracellular effects. Given such unique and excellent characteristics, peptide drugs can be used as novel therapies or replacement therapies ${ }^{4}$. 
Bio-researchers have recently recognized the attractive pharmacological profile of short cationic peptides having significant antibacterial, antifungal, anticancer, and even immunomodulatory activities ${ }^{5-7}$. A report demonstrated that peptides with cation residues (Lysine, Arginine, Histidine) have more significant antimicrobial efficacy than peptides without cation residues ${ }^{8}$. Another study showed that short cationic peptides (SCPs; below six residues) expose better potency than longer peptides. Additionally, SCPs can be synthesized readily by following solid-phase peptide synthesis method ${ }^{9,10}$. A pivotal property of cellpenetrating peptides (CPPs) is their cationic residues, facilitating permeability into the cell membrane ${ }^{11}$. Short peptides with cationic residues (Lysine, Arginine, Histidine) exist essentially in living organisms to function as antimicrobial activity ${ }^{12}$. In animals, antimicrobial peptides (AMPs) are often produced that act as natural innate barriers and elevate immune response to combat microbial infection ${ }^{13-15}$. Interestingly, AMPs have tremendous therapeutic potential to function as antifungal peptides (AFPs) by suppressing the fungal growth such as Candida conidia and hyphae ${ }^{16,17}$. It implies that AMPs play essential roles in boosting the immune system against fungal attack and hence, they are considered new biopharmaceuticals to fight or treat fungal infections. Recent studies have supported that cationic peptides act as immune modulators, recognizing signal molecules like lipopolysaccharide secreted by bacterial or fungal molecules ${ }^{18,19}$.

Evidence also suggests that AMPs demonstrate the antitumor activity by stimulating human cancer cells ${ }^{20}$. The constructed AMPs have positive amino acid residues that can bind effectively with negatively charged cancer cells components ${ }^{21}$. A study proves that AMPs can potentially disrupt the cancer cell membrane due to the strong electrostatic attraction present between positively charged AMPs and negatively charged molecule "phosphatidylserine" on cancer cells' plasma membranes ${ }^{22}$. Another report supports that AMPs activate the host immune defense system, working as anticancer peptides (ACPs) ${ }^{23}$. Despite these advantages, peptides have some intrinsic weaknesses, such as high molecular weight, degradability, and low permeability ${ }^{24}$. However, these limitations can be resolved through traditional design of biotherapeutic peptides that are more suitable for use as convenient therapeutics. Multifunctional and useful cell-penetrating peptides offer more therapeutics and diagnostic merit, leading to the development of future medicines with improved target delivery, efficacy, and pharmacokinetic properties. From these points of view, we used diverse multiple putative AMPs (or) AFPs prediction tools to identify potential therapeutic of SCPs. The final peptides of ACPs were selected via public databases and thus completed AMPs-AFPs-ACPs axis on SCPs.

In this study, we performed network pharmacology (NP) concept to achieve the AMPs-AFPs-ACPs axis. $\mathrm{NP}$ is a collective, systemic, and holistic approach to investigate the relation of molecule(s) and target (s), find the optimal molecule(s) on target protein(s), and provide a crucial hint for identifying the mechanism of a potential lead molecule(s) ${ }^{25-27}$. Moreover, Zhang B. et al. described that NP accelerates the decoding TCM (Traditional Chinese Medicine) from an empirical-based therapy to an evidence-based therapy system, which improves modern drug discovery strategies ${ }^{28}$. 
In our study, network pharmacology-based analysis was utilized to investigate triple therapeutic feasibility (AMPs-AFPs-ACPs axis) of SCPs. Firstly, SCPs (N, and C- terminal cationic groups; $\leq 500$ Dalton) were selected via RStudio analysis. Secondly, the physicochemical propensity of selected SCPs was identified via AMPs screening platform, and a hub signaling pathway of AMPs between AMPsrelated targets and host-responsive targets were analyzed. Thirdly, the AFPs screening platform was used to find AFPs from selected AMPs, and a hub signaling pathway of AMPs-AFPs axis was identified between AFPs-related targets and host-responsive targets. Fourthly, AMPs-AFPs-ACPs axis was constructed by retrieving cancer-related targets from public databases. Fifthly, SCPs accepted by AMPsAFPs-ACPs axis and targets on a hub signaling pathway were subjected to perform MDS. Finally, we found (via network pharmacology) a hub signaling of SCPs which might assume to strengthen the host defense system. Figure 1 shows the overall workflow.

\section{Materials And Methods}

\section{Selection of peptides via RStudio}

The standard peptides were selected with positive amino acids (Lysine, Arginine, Histidine) on both terminals ( $\mathrm{N}$-terminal, $\mathrm{C}$-terminal) or less than 500 Dalton. The selection method of these species was based on RStudio.

\section{AMP evaluation and prediction}

The selected peptides were assessed for AMP evaluation utilizing in silico analysis. Firstly, EMBOSS Pepstats (https://www.ebi.ac.uk/Tools/seqstats/emboss_pepstats/) were used to identify the physicochemical properties of peptides. Secondly, aggregation of peptides was filtered with rigor on both PASTA 2.0 (https://protein.bio.unipd.it/) and AGGRESCAN (http://bioinf.uab.es/aggrescan/) ${ }^{56}$. Subsequently, final AMP were selected by ADAM (http://bioinformatics.cs.ntou.edu.tw/adam/svm_tool.html), dbAMP(http://140.138.77.240/ dbamp/), DBAASP $_{\mathrm{v} 3.0}$ (https://dbaasp.org/prediction/general), and MLAMP (http://www.jci-bioinfo.cn/MLAMP) $57-60$

\section{AFP evaluation and prediction}

The final AMPs' sequences with FASTA format were input to Antifp database (https://webs.iiitd.edu.in/raghava/antifp/predict3.php) ${ }^{61}$. The final AFPs were selected by the classifier of AntipDS1_binary_model1, AntipDS1_binary_model2, and AntipDS1_binary_model3.

\section{Conversion of (peptide) sequences into SMILES format}

The sequences of the final selected AMPs and AFPs were converted to SMILES format through Dendrimer Builder (https://dendrimerbuilder.gdb.tools/) ${ }^{62}$. 
Identification of peptide-target networks and microbial related targets on database

Based on SMILES (format), target targets related to selected peptides were extracted from both SEA (http://sea.bkslab.org/) ${ }^{63}$ and STP (http://www.swisstargetprediction.ch/) ${ }^{64}$ with "Homo Sapiens" setting. The overlapping targets in the peptide(s)-target(s) networks between SEA and STP were identified by VENNY 2.1 (https://bioinfogp.cnb.csic.es/tools/venny/). The bacterial responsive targets on human were obtained with "bacterial/germ/bacilli" from both TTD (http://db.idrblab.net/ttd/) ${ }^{65}$ and OMIM (https://www.omim.org/) ${ }^{66}$ databases. After that, the overlapping targets between peptide(s)-target (s) and bacterial responsive targets were identified by VENNY 2.1

(https://bioinfogp.cnb.csic.es/tools/venny/).

\section{Bubble plot of signaling pathway analysis of overlapping targets between peptide-targets and bacterial responsive targets network}

The final overlapping targets (bacterial responsive targets on the human) networks ware visualized by STRING (https://string-db.org/) ${ }^{67}$. A bubble plot of Kyoto Encyclopedia of Genes and Genomes (KEGG) pathway based on the final overlapping targets was constructed by RStudio.

\section{Identification of peptide-targets network and fungal related targets on database}

Based on SMILES, targets associated with selected peptides were identified via both SEA (http://sea.bkslab.org/) and STP (http://www.swisstargetprediction.ch/) with "Homo Sapiens" setting. The overlapping targets in peptide-target network between SEA and STP were identified by VENNY 2.1 (https://bioinfogp.cnb.csic.es/tools/venny/). The fungal targets associated with a human were obtained from both TTD (http://db.idrblab.net/ttd/) and OMIM (https://www.omim.org/), entering as "fungal". The overlapping targets between peptide-target targets and fungal related targets were identified by VENNY 2.1 (https://bioinfogp.cnb.csic.es/tools/venny/).

\section{Bubble plot of signaling pathway analysis of overlapping targets between peptide-targets and fungal responsive targets network}

The final overlapping targets (fungal responsive targets on the human) construction was visualized by STRING (https://string-db.org/). A bubble plot of Kyoto Encyclopedia of Genes and Genomes (KEGG) pathway based on the final overlapping targets was constructed by RStudio.

\section{Identification of peptide-target targets network and cancer-related targets on database}

Based on SMILES, targets associated with selected peptides were identified via both SEA (http://sea.bkslab.org/) and STP (http://www.swisstargetprediction.ch/) with "Homo Sapiens" setting. The cancer-related targets on human were obtained with "cancer/tumor/neoplasia/carcinoma" from TTD (http://db.idrblab.net/ttd/) and OMIM (https://www.omim.org/). The overlapping targets between peptidetargets and cancer-related targets were identified by VENNY 2.1

(https://bioinfogp.cnb.csic.es/tools/venny/). 
Bubble plot of signaling pathway analysis of overlapping targets between peptide-targets and cancerrelated targets

The final overlapping targets (cancer-related targets on the human) construction was visualized by STRING (https://string-db.org/). RStudio constructed a bubble plot of KEGG pathway based on the final overlapping targets.

\section{Preparation for docking of peptide molecules}

The peptide molecules were converted into SMILES format from Dendrimer builder. The converted SMILES were again converted into .pdb format using Open Babel (http://www.cheminfo.org/Chemistry/Cheminformatics/FormatConverter/index.html) ${ }^{68}$. Finally, the converted .pdb peptide was converted into .pdbqt format through Autodock.

\section{Preparation for docking of target proteins and positive controls to compare with final peptides}

Two target proteins of cancer i.e., STAT3 (.pdb ID: 6TLC), NOS2 (.pdb ID: 4NOS) identified from STRING were converted into .pdbqt format (https://www.rcsb.org/) from .pdb format in order to test the affinity of ligands via Autodock (http://autodock.scripps.edu/) ${ }^{69}$. Subsequently, two positive controls i.e., stattic (Pubchem ID: 2779853) for STAT3 and 1400W (Pubchem ID: 1433) for NOS2, were converted into.pdb format from .sdf format to upload on Pymol, and each of two positive controls was converted again into .pdbqt format to measure affinity through Autodock.

\section{Peptide-target proteins docking test}

The final peptides were docked on target proteins, processing autodock 4 by setting-up 4 energy range and 8 exhaustiveness as default to obtain 10 different poses of ligand molecules ${ }^{70}$. The 2D binding interactions were constructed through LigPlot+ v.2.2 (https://www.ebi.ac.uk/thorntonsrv/software/LigPlus/) ${ }^{71}$.

\section{Results}

\section{SCPs under 500 Dalton rule}

The number of 1,833 peptides with two sufficient conditions (positive N, C- terminals amino acid residues, under 500 Dalton) was selected by RStudio analysis. Table 1 displayed the amount ( $\mathrm{Da}$ ) of each amino acid. The selected peptides were enlisted. (Supplementary Table S1).

\section{Physicochemical refinement for AMPs}

The 1,833 peptides were input in EMBOSS Pepstats (https://www.ebi.ac.uk/Tools/seqstats/emboss_pepstats/) on Charge $>0$ or $8 \leq$ Isoelectric Point $\leq 12{ }^{29}$. Secondly, PASTA 2.0 (adjusted to "zero") (https://protein.bio.unipd.it/) was utilized to predict the peptide 
aggregation propensity ${ }^{30}$. Thirdly, peptide aggregation was checked by AGGRESCAN (Na4VSS $\geq-40$, Na4VSS $\leq 60$ ) (http://bioinf.uab.es/aggrescan/) which was based upon aggregation propensity in vitro. Among 1,833 peptides, the number of 236 peptides were selected (Supplementary Table S2). Fourthly, the 236 peptide sequences were input to four platforms including ADAM (http://bioinformatics.cs.ntou.edu.tw/adam/svm_tool.html), dbAMP (http://140.138.77.240/ dbamp/), DBAASP $_{\text {v3.0 }}$ (https://dbaasp.org/prediction/general), and MLAMP (http://www.jci-bioinfo.cn/MLAMP) to discover AMPs. Finally, from the four databases, 197 out of 236 peptides were obtained as suitable for AMPs (Supplementary Table S3).

\section{AMPs-targets identification}

The number of 197 peptides sequences were converted into SMILE format via Dendrimer Builder (https://dendrimerbuilder.gdb.tools/). The SMILE format of peptide sequences was input to SEA (http://sea.bkslab.org/) and STP (http://www.swisstargetprediction.ch/) databases with "Homo Sapiens"setting. Figure 2A showed that the number of 375 and 355 targets associated with the 197 peptides were identified by SEA and STP, respectively (Supplementary Table S4). The number of 242 overlapping targets was also identified from the two databases (Supplementary Table S5). Finally, Figure 2B and Table 2 displayed that the number of 30 targets overlapped between the number of 959 AMPstargets (extracted from TTD and OMIM databases) (Supplementary Table S6) and overlapping 242 targets were selected.

\section{Signaling pathways responsive to bacterial infection on human}

Figure $3 \mathrm{~A}$ exhibited that 13 out of overlapping 30 targets were notably enriched in 11 signaling pathways via KEGG pathway enrichment analysis. Table 3A showed that the detailed description of the 11 signaling was enlisted. Figure 3B displayed that the 13 targets were associated with the number of 197 peptides, and the constructed peptide-targets networks manifested 210 nodes and 1,011 edges. Figure $3 \mathrm{C}$ showed that peptide-targets network analysis via overlapping 30 targets was constructed by STRING, which indicated 30 nodes and 68 edges. Among 11 signaling pathways, inactivation of Rap 1 signaling pathway was identified as a hub signaling pathway through bubble plot. Figure 3D exhibited that among 11 signaling pathways, the Rap1 signaling pathway's targets were SRC, FPR1, and ITGB1, which was constructed with 158 nodes ( 3 targets, 155 peptides) and 216 edges on a size map. Among the 3 targets (SRC, FPR1, and ITGB1), ITGB1 connected to 117 peptides was on the highest degree of value. It implies that ITGB1 played a vital role in Rap1 signaling pathways in host defense systems against bacterial infection.

\section{Physicochemical refinement for AFPs}

The number of 197 peptides (AMPs) were input into AntipDS1_binary_model1, AntipDS1_binary_model2, and AntipDS1_binary_model3 in antifungal peptide screening platform. Thereby, the number of 91 peptides was accepted as AFPs which were defined as AMPs and AFPs with dual-efficacy for enhancement of host defense system (Supplementary Table S7). 


\section{AFPs-targets identification}

The number of 91 peptides sequences was converted to SMILE format via Dendrimer Builder (https://dendrimerbuilder.gdb.tools/). The SMILE format of peptide sequences was input to SEA (http://sea.bkslab.org/) and STP (http://www.swisstargetprediction.ch/) with "Homo Sapiens"setting. The number of 357 and 330 targets were identified from SEA and STP, respectively (Supplementary Table S8). Figure 4A displayed that the number of 218 overlapping targets was selected from the two databases. (Supplementary Table S9). Figure 4B showed that the number of 6 overlapping targets (TPSAB1, PSEN1, PSEN2, DPP4, STAT3, and NOS2) was identified between the number of AFPs- targets (245 targets from TTD and OMIM databases) (Supplementary Table S10) and overlapping 218 targets.

\section{Signaling pathways responsive to fungal infection on human}

Figure 5A showed that 6 targets (TPSAB1, PSEN1, PSEN2, DPP4, STAT3, and NOS2) were connected to 3 signaling pathways via KEGG pathway enrichment analysis. Table $3 \mathrm{~B}$ showed the detailed description of the 3 signaling. The 6 targets (TPSAB1, PSEN1, PSEN2, DPP4, STAT3, and NOS2) were related to the number of 81 peptides (Supplementary Table S11). Figure 5B exhibited the constructed network exposed 87 nodes (81 peptides, 6 targets) and 1,011 edges. Figure 5C displayed that peptide- targets networking analysis via overlapping 6 targets (TPSAB1, PSEN1, PSEN2, DPP4, STAT3, and NOS2) was constructed by STRING, which indicated 6 nodes and 2 edges. Among 3 signaling pathways, activation of Notch signaling pathway was identified as a hub signaling pathway through bubble plot. Figure 5D showed that Notch signaling pathway's targets were both PSEN1 and PSEN2, and their peptides- targets network was constructed on a size map (34 nodes and 45 edges). Among the 4 targets, PSEN1 and PSEN2 were connected to 9 peptides (KLCK, KCLK, KALK, KVLK, KLGGK, KAFK, KFGK, KFSK, and KSFK) which might have more efficacy than any other AFPs. Besides, it implies that both PSEN1 and PSEN2 played an essential role in the Notch signaling pathway, in aspects of the host defense system against fungal infection on AMPs-AFPs axis.

\section{Cancer-related targets and ACPs-targets identification}

TTD and OMIM selected the number of 4,247 cancer-related targets (Supplementary Table S12). Figure $6 \mathrm{~A}$ exhibited that the number of 4 out of 6 AFP-responsive targets was overlapped with the 4,247 cancerrelated targets. Figure 6B showed that 2 targets (STAT3 and NOS2) were targeted to only HIF-1 signaling pathway via KEGG pathway enrichment analysis. Table 3C showed the detailed description of the signaling. Figure 6C exhibited that the 2 targets (STAT3 and NOS2) were related to the number of 27 peptides, and the constructed networks revealed 29 nodes (27 peptides, 2 targets) and 27 edges. Figure 6D showed that peptide- targets networking analysis via overlapping 4 targets (PSEN1, DPP4, STAT3, NOS2) was constructed by STRING (6 nodes and 2 edges). Figure 6E exhibited that only two targets (STAT3 and NOS2) are related directly to HIF-1 signaling pathway. Both STAT3 and NOS2 targets were directly associated with HIF-1 signaling pathway, which played a crucial role in defending the cancer attack. The HIF-1 signaling pathway was connected particularly to all AMPs-AFPs-ACPs axis. 


\section{MDS on HIF-1 signaling pathway for host defense system}

The ultimate signaling pathway, HIF-1 signaling pathway, was connected to STAT3 (.pdb ID: 6TLC) and NOS2 (.pdb ID: 4NOS): The number of 8 peptides (KPIK, KPVK, KVPK, HPIK, KAFK, KFGK, KSFK, and KFSK) were targeted to STAT3 target, additionally, the number of 19 peptides (RVVK, HMCK, KMCH, HVTK, KCMH, KIIK, KVIK, KILK, KVLK, KALK, KIVK, KIGK, KAIGK, KIAGK, KAGVK, KAGIK, KAGLK, KIGGK, and KVGGK) were targeted to NOS2 target. Table 4 displayed the physicochemical properties of the 27 peptides. The number of 8 peptides was targeted to STAT3 (.pdb ID: 6TLC) and their priorities are as follows: HPIK (-7.3 kcal/mol); KAFK (-7.1 kcal/mol); KPIK (-7.0 kcal/mol); KPVK (-6.8 kcal/mol); KVPK $(-6.8 \mathrm{kcal} / \mathrm{mol}) ; \mathrm{KFGK}(-6.8 \mathrm{kcal} / \mathrm{mol}) ; \mathrm{KSFK}(-6.7 \mathrm{kcal} / \mathrm{mol}) ;$ and KFSK $(-6.4 \mathrm{kcal} / \mathrm{mol})$. Figure $7 \mathrm{~A}$ showed that "HPIK" peptide was the strongest affinity on STAT3 (.pdb ID: 6TLC) in HIF-1 signaling pathway among 8 peptides. Table 5 displayed its detailed information. Likewise, 19 peptides was targeted to NOS2 (.pdb ID: 4NOS), their priorities are as follows: HVTK $(-6.6 \mathrm{kcal} / \mathrm{mol}) ; \mathrm{KILK}(-6.4 \mathrm{kcal} / \mathrm{mol}) ; \mathrm{KAGVK}$ $(-6.1 \mathrm{kcal} / \mathrm{mol}) ; \operatorname{KIGGK}(-6.0 \mathrm{kcal} / \mathrm{mol}) ; \operatorname{KAGLK}(-5.8 \mathrm{kcal} / \mathrm{mol}) ; \mathrm{KAIGK}(-5.6 \mathrm{kcal} / \mathrm{mol}) ; \mathrm{HMCK}(-5.5$ $\mathrm{kcal} / \mathrm{mol}) ; \mathrm{KIAGK}(-5.5 \mathrm{kcal} / \mathrm{mol}) ; \mathrm{KVIK}(-5.5 \mathrm{kcal} / \mathrm{mol}) ; \mathrm{KALK}(-5.5 \mathrm{kcal} / \mathrm{mol}) ; \mathrm{RVVK}(-5.4 \mathrm{kcal} / \mathrm{mol}) ; \mathrm{KIIK}$ $(-5.4 \mathrm{kcal} / \mathrm{mol}) ; \mathrm{KIVK}(-5.4 \mathrm{kcal} / \mathrm{mol}) ; \mathrm{KMCH}(-5.3 \mathrm{kcal} / \mathrm{mol}) ; \mathrm{KVGGK}(-5.3 \mathrm{kcal} / \mathrm{mol}) ; \mathrm{KCMH}(-5.1$ $\mathrm{kcal} / \mathrm{mol}) ; \mathrm{KVLK}(-5.1 \mathrm{kcal} / \mathrm{mol}) ;$ and KAIGK $(-5.0 \mathrm{kcal} / \mathrm{mol})$. Figure 7B showed that "HVTK" peptide was the strongest affinity on NOS2 (.pdb ID: 4NOS) in HIF-1 signaling pathway among 19 peptides. Table 6 showed its detailed information. This result showed that the uppermost promising peptides to strengthen immune system against cancer were "HPIK" on STAT3 (.pdb ID: 6TLC) and "HVTK" on NOS2 (.pdb ID: 4 NOS).

\section{MDS of positive controls on HIF-1 signaling pathway}

The greatest affinity peptide on STAT3 (.pdb ID: 6TLC) was "HPIK" (-7.3 kcal/mol). A representative inhibitor of STAT3 is stattic (PubChem ID: 2779853), which interrupts the tumor cell growth by inhibiting lymphoma activity ${ }^{31}$. Thus, MDS of stattic (PubChem ID: 2779853) was selected to compare with "HPIK". Consequently, the docking score of stattic (PubChem ID: 2779853) was $-6.1 \mathrm{kcal} / \mathrm{mol}$. The "HPIK" affinity on STAT3 (.pdb ID: 6TLC) was better than stattic (PubChem ID: 2779853). The higher affinity peptide on NOS2 (.pdb ID: 4NOS) was "HVTK" $(-6.6 \mathrm{kcal} / \mathrm{mol})$. A selective inhibitor of NOS2 is $1400 \mathrm{~W}$ (PubChem ID: 1433), which could inhibit U87MG cells (brain tumor cell) ${ }^{32}$. Hence, MDS of $1400 \mathrm{~W}$ (PubChem ID: 1433) was carried out to compare with "HVTK"; subsequently, the docking score of 1400W (PubChem ID: 1433) was $-5.2 \mathrm{kcal} / \mathrm{mol}$.

\section{Discussion}

The SCPs were selected with two rigor criteria: $\leq 500$ Dalton and N-, C-terminal cationic amino acid residues. The number of 1,833 SCPs was identified, and consecutively, 197 peptides (AMPs), 91 peptides (AMPs-AFPs axis), and 59 peptides (AMPs-AFPs-ACPs axis) were selected. The associated SCPs with signaling pathways are as followed: 197 peptides-13 targets (AMPs), 81 peptides-6 targets (AMPs-AFPs axis), and 27 peptides-4 targets (AMPs-AFPs-ACPs axis). It was reported that SCPs have functioned as 
antimicrobial agents and host defense adjuvants ${ }^{33}$. A study suggested that TLR4 is an upregulated representative target in keratitis of bacterial infection, whereas SOD2 is an upregulated representative target in keratitis of fungal infection from Differential Expressed Genes (DEGs) ${ }^{34}$. It entails that host responses against bacterial and fungal attack might induce significant differences in the immune system. Hence, we regarded it as an independent perturbation of the bacterial and fungal infection. A study indicated that AMPs could bind with negatively charged ions (phosphatidylserine) on the cancer cell membrane and trigger the host defense system ${ }^{35}$. Thus, we performed the analysis of AMPs-AFPsACPs axis to investigate potential SCPs for the host immune system.

AMPs-targets network showed that the therapeutic efficacy of host defense system was directly associated with $30 \mathrm{~s}$. The result of the KEGG pathway analysis of 30 targets indicated that 11 signaling pathways were connected to 13 out of 30 targets, suggesting that these signaling pathways were directly related to bacterial infection responses in the human immune system.

The description of the 11 signaling pathways with bacterial infection were briefly discussed as follows: Relaxin signaling pathway: Relaxin prevents inflammatory cytokine-induced by endotoxin in THP-1 (human monocytic cell line), which specializes the immune cells in the period of preterm birth ${ }^{36}$. Glucagon signaling pathway: Glucagon alleviates inflammatory responses of the airway, due to association with the reduction of eosinophils and T lymphocytes by inhibiting TCD4+ cell proliferation ${ }^{37,38}$. Prolactin signaling pathway: Prolactin accelerates secretion of proinflammatory cytokines in peripheral immune cells, modulating the level of responses against pathogens ${ }^{39,40}$. Estrogen signaling pathway: Estrogen increases in the level of expression of AMPs in the host, thereby interrupting bacterial proliferation ${ }^{41}$. Additionally, estrogen-stimulated the expression level of cell-cell junction proteins, thereby intensifying the epithelial rigidity and prohibiting unnecessary loss of outer cells during infection ${ }^{42}$. TNF signaling pathway: Tumor Necrosis Factor (TNF) can induce the recruitment of inflammatory cells and control the mechanism of antimicrobial activities ${ }^{43}$. It implies that TNF can work as a buffer element for immunopotentiation. IL-17 signaling pathway: The knock-out groups of IL-17 are highly susceptible to $K$. pneumonia infection than IL-17 expression groups ${ }^{44}$. AMPK signaling pathway: Activation of AMPK improves host defense system against bacterial infection. Moreover, AMPK is associated with the innate and adaptive immune system ${ }^{45}$. FoxO signaling pathway: Fox01 protein is expressed by a bacterial infection, strengthening the epithelial barrier of host cells and induces the recruitment of Tregs (Regulatory T Cells) to activate antibacterial defense ${ }^{46}$. HIF-1 signaling pathway: HIF-1a activation in the hypoxic condition recruits inflammatory-associated cells such as macrophages, neutrophils, and dendritic cells as well as induces offensive cytokine production under bacterial infection ${ }^{47}$. HIF-1 inhibition can be a good strategy to relieve inflammation level induced by the bacterial attack in aspects of the host immune system. Rap1 signaling pathway: The inactivation of Rap1 in lymphocytes is a representative treatment against inflammatory disorders ${ }^{48}$. On AMPs signaling pathways, the key mechanism might inhibit the Rap1 signaling pathway selected based on the rich factor. 
AMPs-AFPs axis-target networks showed that the therapeutic efficacy of host defense system was directly associated with 6 targets. The result of the KEGG pathway analysis of 6 targets were connected to 3 signaling pathways. Neurotrophin signaling pathway: Inflammation signals in microglial cells induce the secretion of neurotrophin that function as mediators of pain ${ }^{49,50}$. It implies that the neurotrophin signaling pathway's inactivation might modulate inflammatory-related proteins' expression level, thereby resolving host defense-induced inflammation. HIF-1 signaling pathway: The deletion of hypoxiaregulated targets are resistant to fungal infection; more importantly, the low-oxygen condition makes fungal virulence attenuate in murine models ${ }^{51}$. Thus, inactivation of HIF-1 might interrupt the fungal penetration and host immune system. Notch signaling pathway: Notch system plays important roles in Th1 and Th2 cell differentiation, and Notch-mediated immune responses are related to $T$ cell development ${ }^{52}$. It supports that the activation of Notch signaling pathway contributes to enhancing the host defense system. On AMPs-AFPs axis signaling pathways, a key signaling pathway is to activate the Notch signaling pathway which was identified based on the rich factor

AMPs-AFPs-ACPs axis-target networks exhibited that the therapeutic efficacy of host defense system was directly associated with 4 targets. The result of the KEGG pathway analysis on 4 targets was connected to 1 signaling pathway. HIF-1 signaling pathway: HIF-1 overexpression contributes to tumor growth, angiogenesis, and metastasis. However, the overexpression is caused by an oxygen-depleted condition in tumor cells ${ }^{53,54}$. Furthermore, hypoxia makes severe conditions under resistance to cancer therapy such as radiation and medication, increasing tumor survival ${ }^{55}$. It suggests that inactivation of a HIF-1 signaling pathway is an optimal strategy for cancer therapy. This work has been focused on immunomodulatory activities of SCPs, which may improve immune defenses and provide key therapeutic agents from large-scale peptides. We have performed the MDT to select promising peptide candidate(s) on the HIF-1 signaling pathway, and hence the standard molecules (static and 1400w) were compared with them. Moreover, we have suggested a hub signaling pathway (HIF-1 signaling pathway), two key SCPs (HPIK and HVTK), and two key targets (STAT3 and NOS2). This analysis collectively suggested an overlapping signaling pathway "HIF-1 signaling pathway" on AMPs, AMPs-AFPs axis, and AMPs-AFPsACPs axis. Therefore, the inactivation of HIF-1 signaling pathway using two selected peptides is a feasible treatment strategy for enhancing the host defense system.

\section{Conclusion}

The uppermost SCPs of AMPs-AFPs-ACPs axis for immunopotentiation were firstly investigated through network pharmacology. The number of 1,833 SCPs was funneled sequentially through peptide screening platform, thereby, the number of 197 SCPs (AMPs), and 91 SCPs (AMPs-AFPs axis) were obtained. The number of 27 SCPs (AMPs-AFPs-ACPs axis) was obtained as final promising peptides through cancerrelated targets analysis. The 27 SCPs (AMPs-AFPs-ACPs axis) were connected to only the HIF-1 signaling pathway with HPIK-STAT3 and HVTK-NOS2. This analysis provides the network of two SCPs, two targets, and one signaling pathway for the host defense system. Consequently, the key findings on AMPs-AFPsACPs axis could be a promising therapeutic strategy for cellular protection against immune disorders.

Page $11 / 34$ 


\section{Abbreviations}

ACPs: Anticancer peptides;

AFPs: Antifungal peptides;

AMPs: Antimicrobial peptides;

AMPK: AMP-activated protein kinase;

DEGs: Differential Expressed Genes;

FDA: Food and drug administration;

FoxO: Forkhead box 0;

HIF-1: Hypoxia Inducible Factor 1;

KEGG: Kyoto Encyclopedia of Genes and Genomes;

MDS: Molecular Docking Study;

Rap1: Repressor Activator Protein;

SCPs: Short cationic peptides;

SEA: Similarity Ensemble Approach;

SMILES: Simplified Molecular Input Line Entry System;

STP: SwissTargetPrediction;

TCM: Traditional Chinese Medicine;

TNF: Tumor Necrosis Factor;

Tregs: Regulatory T cells

\section{Declarations}

\section{Data availability}

All data generated or analysed during this study are included in this published article (and its Supplementary Information files).

Acknowledgements This research was acknowledged by the Department of Bio-Health Convergence, College of Biomedical Science, Kangwon National University, Chuncheon, 24341, Korea. 


\section{Author contributions}

K.K.O.: Conceptualization, Methodology, Formal analysis, Visualization, Investigation, Software, Data Curation, Writing-Original Draft. K.K.O. and M.A.: Investigation, Data Curation. M.A.: Validation, WritingReview \& Editing. D.H.C.: Supervision, Project administration.

\section{Competing interests}

The authors declare no competing interests.

\section{References}

1. Uhlig, T. et al. The emergence of peptides in the pharmaceutical business: From exploration to exploitation. EuPA Open Proteomics 4, 58-69 (2014).

2. Lau, J. L. \& Dunn, M. K. Therapeutic peptides: Historical perspectives, current development trends, and future directions. Bioorganic and Medicinal Chemistry 26, 2700-2707 (2018).

3. Angell, Y., Holford, M. \& Moos, W. H. Building on Success: A Bright Future for Peptide Therapeutics. Protein \& Peptide Letters 25, 1044-1050 (2018).

4. Fosgerau, K. \& Hoffmann, T. Peptide therapeutics: current status and future directions. Drug discovery today 20, 122-128 (2015).

5. Bhat, Z. F., Kumar, S. \& Bhat, H. F. Bioactive peptides of animal origin: a review. Journal of Food Science and Technology vol. 52 5377-5392 (2015).

6. Mishra, B. et al. Antibacterial, antifungal, anticancer activities and structural bioinformatics analysis of six naturally occurring temporins. Peptides 106, 9-20 (2018).

7. Haney, E. F. \& Hancock, R. E. W. Peptide design for antimicrobial and immunomodulatory applications. Biopolymers vol. 100 572-583 (2013).

8. Bormann, N. et al. A short artificial antimicrobial peptide shows potential to prevent or treat bone infections. Scientific Reports 7, 1-14 (2017).

9. Kuppusamy, R., Willcox, M., Black, D. S. C. \& Kumar, N. Short cationic peptidomimetic antimicrobials. Antibiotics vol. 8 (2019).

10. Wenzel, M. et al. Small cationic antimicrobial peptides delocalize peripheral membrane proteins. Proceedings of the National Academy of Sciences of the United States of America 111, E1409-E1418 (2014).

11. Ziegler, A., Nervi, P., Dürrenberger, M. \& Seelig, J. The cationic cell-penetrating peptide CPPTAT derived from the HIV-1 protein TAT is rapidly transported into living fibroblasts: Optical, biophysical, and metabolic evidence. Biochemistry 44, 138-148 (2005).

12. Hancock, R. E. W. \& Lehrer, R. Cationic peptides: A new source of antibiotics. Trends in Biotechnology vol. 16 82-88 (1998). 
13. Brogden, K. A., Ackermann, M., McCray, P. B. \& Tack, B. F. Antimicrobial peptides in animals and their role in host defences. International Journal of Antimicrobial Agents vol. 22 465-478 (2003).

14. Oppenheim, J. J. \& Yang, D. Alarmins: Chemotactic activators of immune responses. Current Opinion in Immunology vol. 17 359-365 (2005).

15. Bowdish, D., Davidson, D. \& Hancock, R. A Re-evaluation of the Role of Host Defence Peptides in Mammalian Immunity. Current Protein \& Peptide Science 6, 35-51 (2005).

16. van Eijk, M. et al. Cathelicidin-inspired antimicrobial peptides as novel antifungal compounds. Medical Mycology 58, 1073-1084 (2020).

17. Netea, M. G., Joosten, L. A. B., Van Der Meer, J. W. M., Kullberg, B. J. \& Van De Veerdonk, F. L. Immune defence against Candida fungal infections. Nature Reviews Immunology vol. 15 630-642 (2015).

18. Hancock, R. E. W. \& Sahl, H. G. Antimicrobial and host-defense peptides as new anti-infective therapeutic strategies. Nature Biotechnology vol. 24 1551-1557 (2006).

19. Finlay, B. B. \& Hancock, R. E. W. Can innate immunity be enhanced to treat microbial infections? Nature Reviews Microbiology 2, 497-504 (2004).

20. Deslouches, B. \& Peter Di, Y. Antimicrobial peptides with selective antitumor mechanisms: Prospect for anticancer applications. Oncotarget vol. 8 46635-46651 (2017).

21. Gaspar, D., Salomé Veiga, A. \& Castanho, M. A. R. B. From antimicrobial to anticancer peptides. A review. Frontiers in Microbiology vol. 4294 (2013).

22. Digumarti, R. et al. Bavituximab plus paclitaxel and carboplatin for the treatment of advanced nonsmall-cell lung cancer. Lung Cancer 86, 231-236 (2014).

23. Guzmán-Rodríguez, J. J., Ochoa-Zarzosa, A., López-Gómez, R. \& López-Meza, J. E. Plant antimicrobial peptides as potential anticancer agents. BioMed Research International vol. 2015 (2015).

24. Lee, A. C. L., Harris, J. L., Khanna, K. K. \& Hong, J. H. A comprehensive review on current advances in peptide drug development and design. International Journal of Molecular Sciences vol. 20 (2019).

25. Oh, K. K., Adnan, M. \& Cho, D. H. Active ingredients and mechanisms of Phellinus linteus (grown on Rosa multiflora) for alleviation of Type 2 diabetes mellitus through network pharmacology. Gene (2020) doi:10.1016/j.gene.2020.145320.

26. Oh, K. K., Adnan, M. \& Cho, D. H. Network pharmacology approach to bioactive chemical compounds identified from Lespedeza bicolor lignum methanol extract by GC-MS for amelioration of hepatitis. Gene Reports 100851 (2020).

27. Oh, K. K., Adnan, M. \& Cho, D. H. Network pharmacology of bioactives from Sorghum bicolor with targets related to diabetes mellitus. PLOS ONE 15, e0240873 (2020).

28. Li, S. \& Zhang, B. Traditional Chinese medicine network pharmacology: Theory, methodology and application. Chinese Journal of Natural Medicines 11, 110-120 (2013).

29. Lee, J. H. et al. Transcriptome analysis of psacothea hilaris: De novo assembly and antimicrobial peptide prediction. Insects 11, 1-11 (2020). 
30. Walsh, I., Seno, F., Tosatto, S. C. E. \& Trovato, A. PASTA 2.0: An improved server for protein aggregation prediction. Nucleic Acids Research 42, (2014).

31. Scuto, A. et al. STAT3 inhibition is a therapeutic strategy for ABC-like diffuse large B-cell lymphoma. Cancer Research 71, 3182-3188 (2011).

32. Palumbo, P. et al. NOS2 inhibitor 1400W induces autophagic flux and influences extracellular vesicle profile in human glioblastoma U87MG cell line. International Journal of Molecular Sciences 20, (2019).

33. Brown, K. L. \& Hancock, R. E. W. Cationic host defense (antimicrobial) peptides. Current Opinion in Immunology vol. 18 24-30 (2006).

34. Tian, R. et al. Analysis of differentially expressed genes in bacterial and fungal keratitis. Indian Journal of Ophthalmology 68, 39-46 (2020).

35. Deslouches, B. \& Peter Di, Y. Antimicrobial peptides with selective antitumor mechanisms: Prospect for anticancer applications. Oncotarget vol. 8 46635-46651 (2017).

36. Horton, J. S., Yamamoto, S. Y. \& Bryant-Greenwood, G. D. Relaxin modulates proinflammatory cytokine secretion from human decidual macrophages. Biology of Reproduction 85, 788-797 (2011).

37. Insuela, D. B. R. et al. Glucagon reduces airway hyperreactivity, inflammation, and remodeling induced by ovalbumin. Scientific Reports 9, 1-17 (2019).

38. Long, H., Liao, W., Wang, L. \& Lu, Q. A Player and Coordinator: The Versatile Roles of Eosinophils in the Immune System. Transfusion Medicine and Hemotherapy vol. 43 96-108 (2016).

39. Boutet, P. et al. Prolactin-induced activation of nuclear factor кB in bovine mammary epithelial cells: Role in chronic mastitis. Journal of Dairy Science 90, 155-164 (2007).

40. Yu-Lee, L. Y. Prolactin modulation of immune and inflammatory responses. Recent Progress in Hormone Research 57, 435-455 (2002).

41. Hannan, T. J., Hooton, T. M. \& Hultgren, S. J. Estrogen and recurrent UTI: What are the facts? Science Translational Medicine vol. 5 190fs23-190fs23 (2013).

42. Lüthje, P. et al. Estrogen supports urothelial defense mechanisms. Science Translational Medicine 5, 190ra80-190ra80 (2013).

43. Ehlers, S. Role of tumour necrosis factor (TNF) in host defence against tuberculosis: implications for immunotherapies targeting TNF. Annals of the Rheumatic Diseases 62, 37ii - 42 (2003).

44. Qian, Y., Kang, Z., Liu, C. \& Li, X. IL-17 signaling in host defense and inflammatory diseases. Cellular and Molecular Immunology vol. 7 328-333 (2010).

45. Silwal, P., Kim, J. K., Yuk, J. M. \& Jo, E. K. AMP-Activated protein kinase and host defense against infection. International Journal of Molecular Sciences vol. 19 (2018).

46. Graves, D. T. \& Milovanova, T. N. Mucosal Immunity and the FOX01 Transcription Factors. Frontiers in Immunology vol. 102530 (2019). 
47. Palazon, A., Goldrath, A. W., Nizet, V. \& Johnson, R. S. HIF Transcription Factors, Inflammation, and Immunity. Immunity vol. 41 518-528 (2014).

48. Mor, A., Haklai, R., Ben-Moshe, O., Mekori, Y. A. \& Kloog, Y. Inhibition of contact sensitivity by farnesylthiosalicylic acid-amide, a potential rap1 inhibitor. Journal of Investigative Dermatology 131, 2040-2048 (2011).

49. Heese, K., Hock, C. \& Otten, U. Inflammatory signals induce neurotrophin expression in human microglial cells. Journal of Neurochemistry 70, 699-707 (1998).

50. Mannion, R. J. et al. Neurotrophins: Peripherally and centrally acting modulators of tactile stimulusinduced inflammatory pain hypersensitivity. Proceedings of the National Academy of Sciences of the United States of America 96, 9385-9390 (1999).

51. Grahl, N., Shepardson, K. M., Chung, D. \& Cramer, R. A. Hypoxia and fungal pathogenesis: To air or not to air? Eukaryotic Cell vol. 11 560-570 (2012).

52. Ito, T., Connett, J. M., Kunkel, S. L. \& Matsukawa, A. Notch system in the linkage of innate and adaptive immunity. Journal of Leukocyte Biology 92, 59-65 (2012).

53. Burroughs, S. K. et al. Hypoxia inducible factor pathway inhibitors as anticancer therapeutics. Future Medicinal Chemistry vol. 5 553-572 (2013).

54. Semenza, G. L. Targeting HIF-1 for cancer therapy. Nature Reviews Cancervol. 3 721-732 (2003).

55. Jing, X. et al. Role of hypoxia in cancer therapy by regulating the tumor microenvironment. Molecular Cancervol. 18 (2019).

56. Lee, J. H. et al. Transcriptome analysis of psacothea hilaris: De novo assembly and antimicrobial peptide prediction. Insects (2020) doi:10.3390/insects11100676.

57. Lee, H.-T., Lee, C.-C., Yang, J.-R., Lai, J. Z. C. \& Chang, K. Y. A Large-Scale Structural Classification of Antimicrobial Peptides. BioMed Research International 2015, 1-6 (2015).

58. Wang, G. The antimicrobial peptide database provides a platform for decoding the design principles of naturally occurring antimicrobial peptides. Protein Science 29, 8-18 (2020).

59. Gogoladze, G. et al. DBAASP: Database of antimicrobial activity and structure of peptides. FEMS Microbiology Letters 357, 63-68 (2014).

60. Lin, W. \& Xu, D. Imbalanced multi-label learning for identifying antimicrobial peptides and their functional types. Bioinformatics 32, 3745-3752 (2016).

61. Agrawal, P. et al. In Silico Approach for Prediction of Antifungal Peptides. Frontiers in Microbiology 9 , 323 (2018).

62. Maingi, V., Jain, V., Bharatam, P. V. \& Maiti, P. K. Dendrimer building toolkit: Model building and characterization of various dendrimer architectures. Journal of Computational Chemistry 33, 19972011 (2012).

63. Keiser, M. J. et al. Relating protein pharmacology by ligand chemistry. Nature Biotechnology 25, 197-206 (2007). 
64. Daina, A., Michielin, O. \& Zoete, V. SwissTargetPrediction: updated data and new features for efficient prediction of protein targets of small molecules. Nucleic Acids Research 47, W357-W3664 (2019).

65. Wang, Y. et al. Therapeutic target database 2020: Enriched resource for facilitating research and early development of targeted therapeutics. Nucleic Acids Research 48, D1031-D1041 (2020).

66. Amberger, J. S., Bocchini, C. A., Scott, A. F. \& Hamosh, A. OMIM.org: Leveraging knowledge across phenotype-gene relationships. Nucleic Acids Research 47, D1038-D1043 (2019).

67. Szklarczyk, D. et al. STRING v11: Protein-protein association networks with increased coverage, supporting functional discovery in genome-wide experimental datasets. Nucleic Acids Research 47, D607-D613 (2019).

68. O'Boyle, N. M. et al. Open Babel: An Open chemical toolbox. Journal of Cheminformatics 3,1-14 (2011).

69. Morris, G. M. et al. Software news and updates AutoDock4 and AutoDockTools4: Automated docking with selective receptor flexibility. Journal of Computational Chemistry 30, 2785-2791 (2009).

70. Khanal, P., Patil, B. M., Chand, J. \& Naaz, Y. Anthraquinone Derivatives as an Immune Booster and their Therapeutic Option Against COVID-19. Natural Products and Bioprospecting 10, 325-335 (2020).

71. Laskowski, R. A. \& Swindells, M. B. LigPlot+: Multiple ligand-protein interaction diagrams for drug discovery. Journal of Chemical Information and Modeling 51, 2778-2786 (2011).

\section{Tables}

Table 1. The amount (Da) of 20 amino acids. 


\begin{tabular}{ll} 
Amino acid & Residue mass (DA) \\
\hline A & 71.08 \\
\hline R & 156.2 \\
\hline N & 114.11 \\
\hline D & 115.09 \\
\hline C & 103.14 \\
\hline Q & 128.14 \\
\hline E & 129.12 \\
\hline G & 57.06 \\
\hline$H$ & 137.15 \\
\hline I & 113.17 \\
\hline L & 113.17 \\
\hline K & 128.18 \\
\hline M & 131.21 \\
\hline F & 147.18 \\
\hline P & 97.12 \\
\hline S & 87.08 \\
\hline T & 101.11 \\
\hline W & 186.21 \\
\hline Y & 163.18 \\
\hline V & 99.14 \\
\hline
\end{tabular}

A: Alanine; R: Arginine; N: Asparagine; D: Aspartic acid; C: Cysteine; Q: Glutamine; E: Glutamic acid; G: Glycine; H: Histidine; I: Isoleucine; L: Leucine; K: Lysine; M: Methionine; F: Phenylalanine; P: Proline; S: Serine; T: Threonine; W: Tryptophan; Y: Tyrosine; V: Valine

Table 2. The number of 30 targets overlapped between 959 AMPs-targets and overlapping 242 targets. 


\begin{tabular}{clll} 
No. & Targets & No. & Targets \\
\hline 1 & ACE & 16 & CA2 \\
\hline 2 & ECE1 & 17 & ITGB1 \\
\hline 3 & EDNRA & 18 & GLO1 \\
\hline 4 & MMP3 & 19 & MC1R \\
\hline 5 & SIRT1 & 20 & OPRM1 \\
\hline 6 & SIRT2 & 21 & PPARG \\
\hline 7 & TPP2 & 22 & PYGL \\
\hline 8 & UBE2I & 23 & SRC \\
\hline 9 & CASP1 & 24 & PLAU \\
\hline 10 & FPR1 & 25 & ELANE \\
\hline 11 & MMP9 & 26 & STAT3 \\
\hline 12 & PDYN & 27 & NOS2 \\
\hline 13 & MMP12 & 28 & GLUL \\
\hline 14 & SIRT3 & 29 & DHFR \\
\hline 15 & PDF & 30 & ITGA5 \\
\hline
\end{tabular}

Table 3(A). Targets in 11 signaling pathways enrichment related to AMPs. 


\begin{tabular}{clll} 
KEGG ID & Description & Targets & False discovery rate \\
\hline hsa04917 & Prolactin signaling pathway & SRC,STAT3 & 0.0283 \\
\hline hsa04926 & Relaxin signaling pathway & SRC,NOS2,MMP9 & 0.0093 \\
\hline hsa04915 & Estrogen signaling pathway & SRC,OPRM1,MMP9 & 0.0093 \\
\hline hsa04657 & IL-17 signaling pathway & MMP3,MMP9 & 0.0359 \\
\hline hsa04064 & NF-kappa B signaling pathway & PLAU,UBE2I & 0.0359 \\
\hline hsa04066 & HIF-1 signaling pathway & STAT3,NOS2 & 0.0359 \\
\hline hsa04922 & Glucagon signaling pathway & SIRT1,PYGL & 0.0360 \\
\hline hsa04668 & TNF signaling pathway & MMP3,MMP9 & 0.0389 \\
\hline hsa04152 & AMPK signaling pathway & SIRT1,PPARG & 0.0448 \\
\hline hsa04068 & FoxO signaling pathway & STAT3,SIRT1 & 0.0496 \\
\hline hsa04015 & Rap1 signaling pathway & SRC,ITGB1,FPR1 & 0.0243
\end{tabular}

Table 3(B). Targets in 3 signaling pathways enrichment related to AMPs-AFPs axis.

\begin{tabular}{llll} 
KEGG ID & Description & Targets & False discovery rate \\
\hline hsa04330 & Notch signaling pathway & PSEN1,PSEN2 & 0.0044 \\
\hline hsa04066 & HIF-1 signaling pathway & NOS2,STAT3 & 0.0088 \\
\hline hsa04722 & Neurotrophin signaling pathway & PSEN1,PSEN2 & 0.0088
\end{tabular}

Table 3(C). Targets in 1 signaling pathway enrichment related to AMPs-AFPs-ACPs axis.

\begin{tabular}{llll} 
KEGG ID & Description & Targets & False discovery rate \\
\hline hsa04066 & HIF-1 signaling pathway & NOS2,STAT3 & 0.0071
\end{tabular}

Table 4. The physicochemical properties of final 27 peptides on AMPs-AFPs-ACPs axis. 
No. Peptide sequence
Residue mass

(Da)

\begin{tabular}{|c|c|c|c|c|c|c|}
\hline & & $(\mathrm{Da})$ & & $(>0)$ & $(8 \leq ; \geq 12)$ & $\begin{array}{l}\text { (Na4VSS } \geq-40 ; \\
\text { Na4VSS } \leq 60)\end{array}$ \\
\hline 1 & KPIK & 466.65 & Nos2 & 2 & 10.8 & -34.6 \\
\hline 2 & KPVK & 452.62 & NOS2 & 2 & 10.8 & -39.1 \\
\hline 3 & KVPK & 452.62 & NOS2 & 2 & 10.8 & -39.1 \\
\hline 4 & HPIK & 475.62 & NOS2 & 1.5 & 9.2 & -36.6 \\
\hline 5 & KAFK & 474.62 & NOS2 & 2 & 10.8 & -30.0 \\
\hline 6 & KFGK & 460.60 & NOS2 & 2 & 10.8 & -40.0 \\
\hline 7 & KSFK & 490.62 & NOS2 & 2 & 10.8 & -35.1 \\
\hline 8 & KFSK & 490.62 & Nos2 & 2 & 10.8 & -35.1 \\
\hline 9 & RVVK & 482.66 & STAT3 & 2 & 11.7 & -6.7 \\
\hline 10 & HMCK & 499.68 & STAT3 & 1.5 & 8.0 & -36.1 \\
\hline 11 & $\mathrm{KMCH}$ & 499.68 & STAT3 & 1.5 & 8.0 & -36.1 \\
\hline 12 & HVTK & 465.58 & STAT3 & 1.5 & 9.2 & -37.7 \\
\hline 13 & $\mathrm{KCMH}$ & 499.68 & STAT3 & 1.5 & 8.0 & -36.1 \\
\hline 14 & KIIK & 482.70 & STAT3 & 2 & 10.8 & 8.6 \\
\hline 15 & KVIK & 468.67 & STAT3 & 2 & 10.8 & 4.0 \\
\hline 16 & KILK & 482.70 & STAT3 & 2 & 10.8 & -0.3 \\
\hline 17 & KVLK & 468.67 & STAT3 & 2 & 10.8 & -4.8 \\
\hline 18 & KALK & 440.61 & STAT3 & 2 & 10.8 & -37.4 \\
\hline 19 & KIVK & 468.67 & STAT3 & 2 & 10.8 & 4.0 \\
\hline 20 & KIGK & 426.59 & STAT3 & 2 & 10.8 & -38.6 \\
\hline 21 & KAIGK & 497.57 & STAT3 & 2 & 10.8 & -19.0 \\
\hline 22 & KIAGK & 497.57 & STAT3 & 2 & 10.8 & -19.0 \\
\hline 23 & KAGVK & 483.64 & STAT3 & 2 & 10.8 & -23.6 \\
\hline 24 & KAGIK & 497.67 & STAT3 & 2 & 10.8 & -19.0 \\
\hline 25 & KAGLK & 497.67 & STAT3 & 2 & 10.8 & -27.8 \\
\hline 26 & KIGGK & 483.65 & STAT3 & 2 & 10.8 & -29.0 \\
\hline
\end{tabular}

Targets Charge Isoelectric Aggregation propensity point 
Table 5. Binding energy and interactions of potential active SCPs and standard molecule (stattic) on STAT3 (PDB ID: 6TLC). 
Hydrogen Bond

Interactions

Peptide sequence

HPIK

(

KAFK

$-7.1$

Binding energy $(\mathrm{kcal} / \mathrm{mol})$

$-7.3$

GIn361,Tyr446

Amino Acid Residue

Hydrophobic Interactions

Amino Acid Residue

Gln448,Glu444,Leu358,

Glu357,His447,Gln448,

KAFK

KPIK

$-7.0$

$-6.8$

KPVK

KVPK

$-6.8$

Tyr446

Gln361

Glu444,Tyr446,GIn361,

Gln448,Glu357,Gln448,

\section{Leu362}

Gln448,Glu357,Leu358,

Gly449,Val445

His447,

Glu357,Gln448,

Tyr446,Gly449,Val445

KFGK

$-6.8$

Glu306,Arg278,Lys282,

$\mathrm{G} \ln 361, \mathrm{G} \ln 448$

Ile309,Tyr360,Lys283,

GIn279,Glu286,Leu362,

Lys363,Leu450,Gly449,

Val310

KSFK

$-6.7$

$-6.4$

KFSK

$-6.4$

Binding
energy (kcal/mol)

Compound

(PubChem ID)

Stattic (2779853)
$-6.1$
Lys363,Gly449,GIn448, Glu444,His447,Tyr446,

Tyr446,GIn361,Thr443 His447,Glu357,GIn448,

Val445,Glu357,Leu358,

Tyr446,Gly449,GIn361, Gln448,Glu444,Val445,

Gln448

His447,Glu357,His447,

Glu357

Hydrogen Bond

Interactions

Hydrophobic

Interactions

Amino Acid Residue

Amino Acid Residue

Gly449,Tyr446,GIn361

Gln448,Tyr446,Glu357, 
Table 6. Binding energy and interactions of potential active SCPs and standard molecule (1400W) on NOS2 (PDB ID: 4NOS). 
Hydrogen Bond

Interactions

\section{Peptide sequence}

HVTK

HVTK

KILK

$-6.4$

KAGVK

KAGLK

KAIGK

$-5.5$

KVIK

Binding

$-6.6$

$-6.1$

$-6.0$

$-5.8$

$-5.6$

$-5.5$

Arg454,Ser453

Glu145

Asn196,Arg454 energy $(\mathrm{kcal} / \mathrm{mol}$ )

Glu145,GIn192,Arg195

Arg454,Ser486,GIn192

Ser153,Gln192,Asn196,

Glu450,Arg454,Asn196, Ser453,Trp206,Leu100,

Lys103,Gln192,Arg195 Leu485,Ser486,Gly152,

GIn149,Ser153

GIn149,GIn192,Asn148, Arg454,Arg195,Gly275,

Asp274,Pro273,Gly152,

Ser486,Lys103,Phe188,

Leu485,Leu100

Gln149,Lys103,Leu485,

Leu100,Glu145,Asn148,

Gly152

Gln192,Ser486,Glu245 Ser153,Gly152,Arg195,

Lys103,Leu485,Gln149,

Leu100,Ser453

Lys103,Leu100,Leu485,

Arg454,Gln149,Asn148

Glu145,Asn196,Gln192, Ser486,Leu485,Gln149, 


\begin{tabular}{|c|c|c|c|}
\hline & & Arg454 & Asn148,Pro273,Lys103 \\
\hline \multirow[t]{3}{*}{ KALK } & -5.5 & Ser486,GIn149,Asp274 & Leu100,Gly152,Pro273, \\
\hline & & & Asn148,Glu145,Lys103, \\
\hline & & & Leu485 \\
\hline \multirow[t]{3}{*}{ RVVK } & -5.4 & $\mathrm{G} \ln 192$ & Ser153,Arg454,GIn149, \\
\hline & & & Gly152,Asp274,Asn148, \\
\hline & & & Lys103,Leu485,Leu100 \\
\hline \multirow[t]{3}{*}{ KIIK } & -5.4 & Lys103,GIn192,Arg195 & Leu485,GIn149,Leu100, \\
\hline & & & Ser453,Ser153,Gly152, \\
\hline & & & Glu145,Ser486 \\
\hline \multirow[t]{3}{*}{ KIVK } & -5.4 & Pro273,An148,Glu145 & Ser486,Lys103,Leu485, \\
\hline & & & Asn196,Leu100,Arg454, \\
\hline & & & Gln149,Gly152 \\
\hline \multirow[t]{3}{*}{$\mathrm{KMCH}$} & -5.3 & Arg195,Gln192,Ser486, & Ser153,Asp274,Asn148, \\
\hline & & Gln149,Lys103 & Gly275,Pro273,Glu145, \\
\hline & & & Leu100,Arg454 \\
\hline \multirow[t]{4}{*}{ KVGGK } & -5.3 & Thr121,Arg86,Thr126 & Trp90,Glu479,Ile119, \\
\hline & & & Val85,Arg83,His84, \\
\hline & & & Leu116,Thr109,Pro122, \\
\hline & & & Lys123 \\
\hline \multirow[t]{3}{*}{ KIGK } & -5.3 & Gly152,Lys103,Glu145, & Asn148,Leu485,Ser486, \\
\hline & & Asn196,Arg454 & Leu100,GIn149,Ser453 \\
\hline & & & Ser153 \\
\hline \multirow[t]{2}{*}{$\mathrm{KCMH}$} & -5.1 & Lys103,Gln149,Gln192 & Ser453,Gly152,Leu100, \\
\hline & & & Leu485,Ser486,Ser153 \\
\hline KVLK & -5.1 & Ile277,Asn390,Gly279 & Arg278,Ser276,Leu344, \\
\hline
\end{tabular}


Arg301,Ile391,Pro281,

Tyr389,Arg388

KAGIK

$-5.0$

Asn196

Arg454,Arg195,Ser153,

Leu100,Lys103,Ser486,

Leu485,Glu145,Asn148,

GIn149,Gly152,GIn192

Hydrogen Bond

Interactions

Amino Acid Residue

energy $(\mathrm{kcal} / \mathrm{mol})$

(PubChem ID)

1400W(1433)
$-5.2$
Gln97

Gly455,Arg452,Tyr451,
Hydrophobic
Interactions

\section{Amino Acid Residue}

Met94,GIn448,Thr95,

Phe96

\section{Figures}

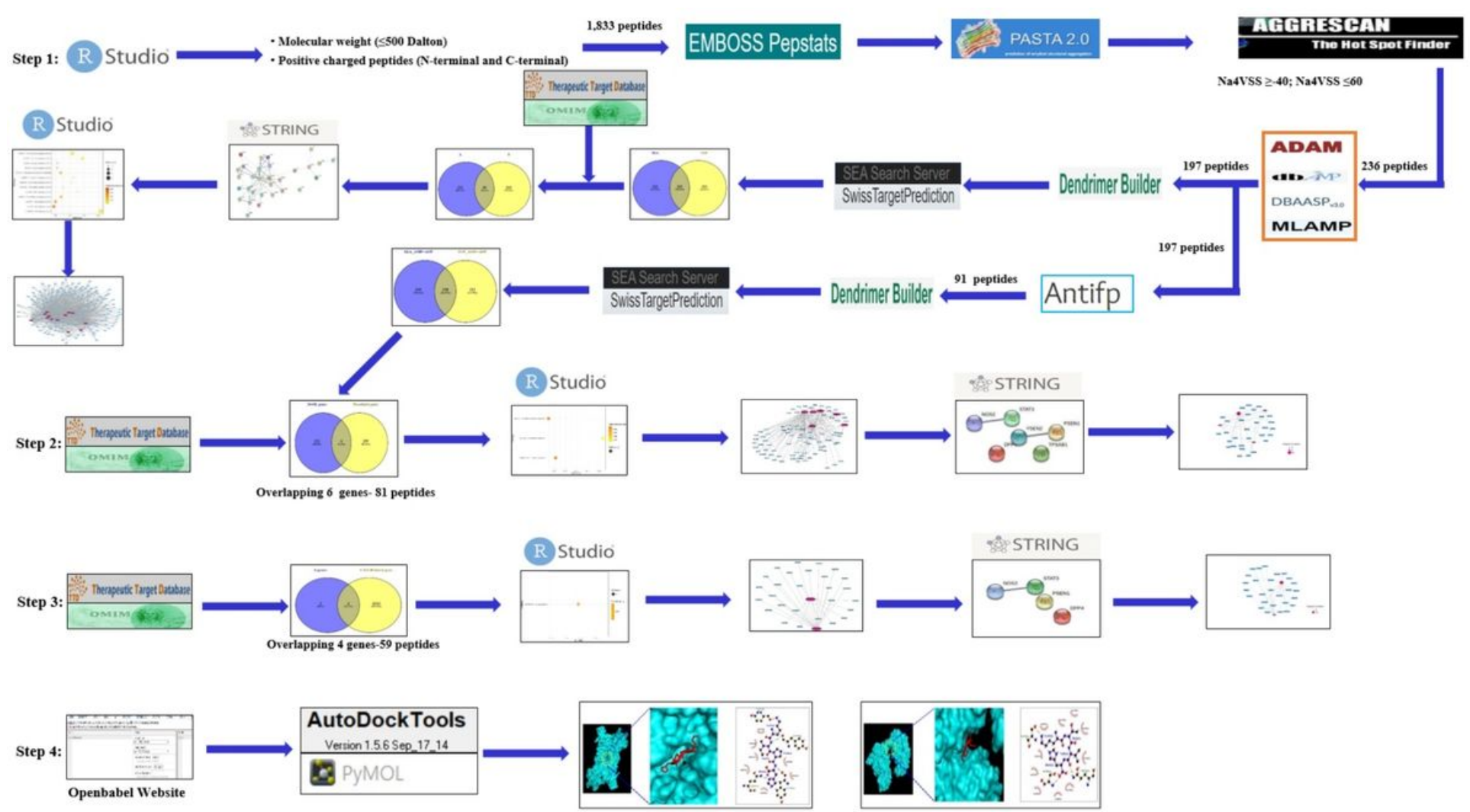

Figure 1

Workflow of AMPs-AFPs-ACPs axis analysis on network pharmacology. 
(A)

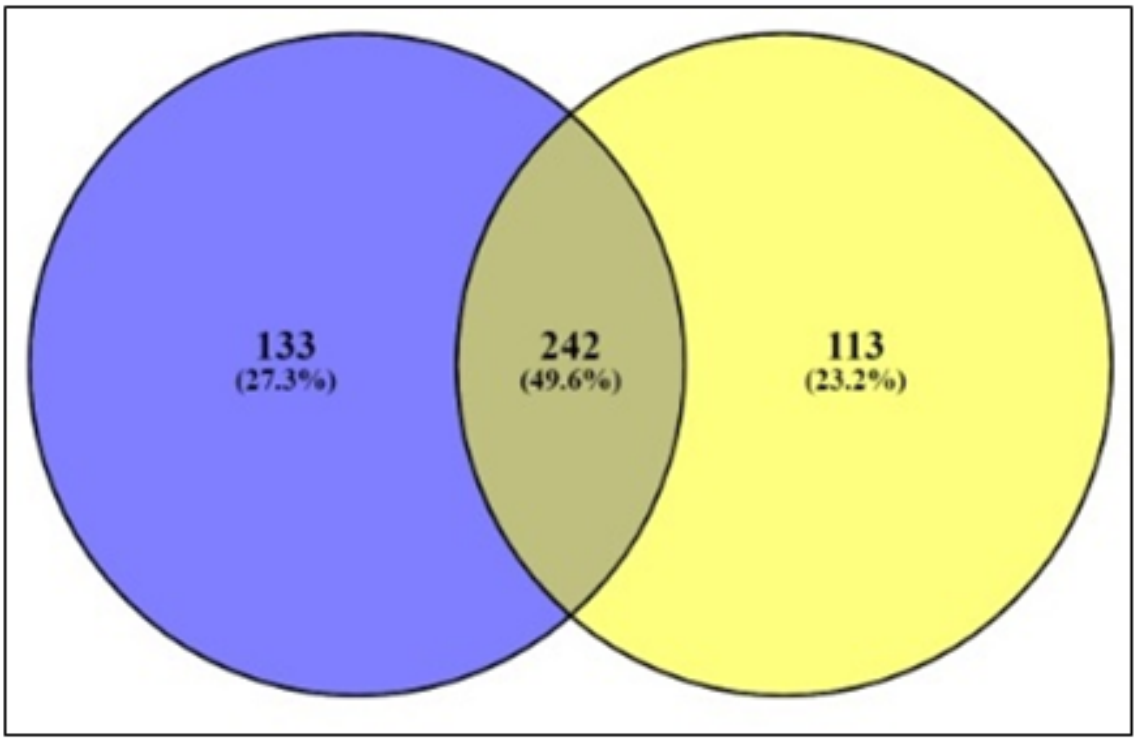

(B)

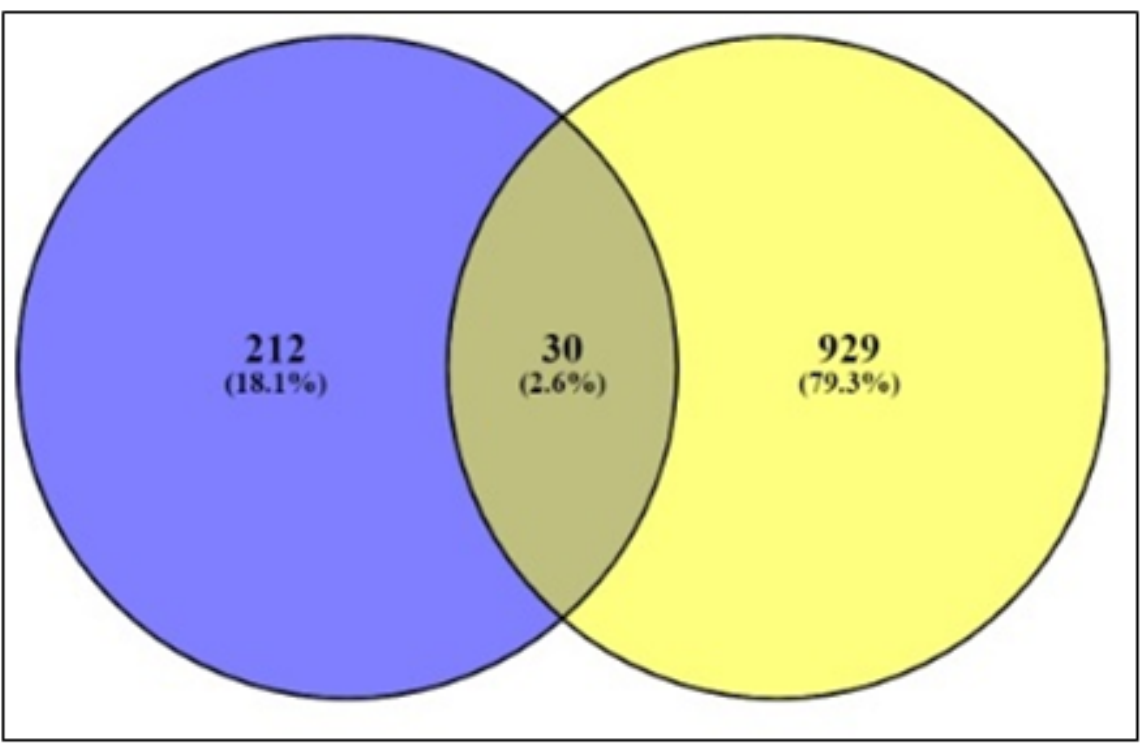

Figure 2

(A) The number of 242 overlapping targeted targets from SEA (375 targets) and STP (355 targets) on AMPs- targets. (B) The number of 30 overlapping targets between the number of 242 overlapping targets and 959 bacterial respond targets. 
(A)

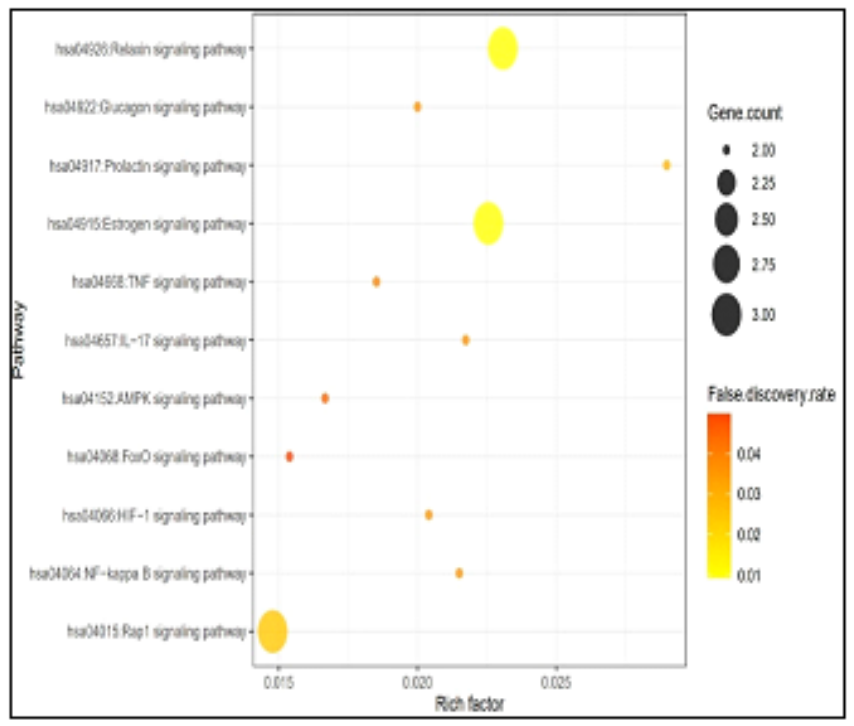

(C)

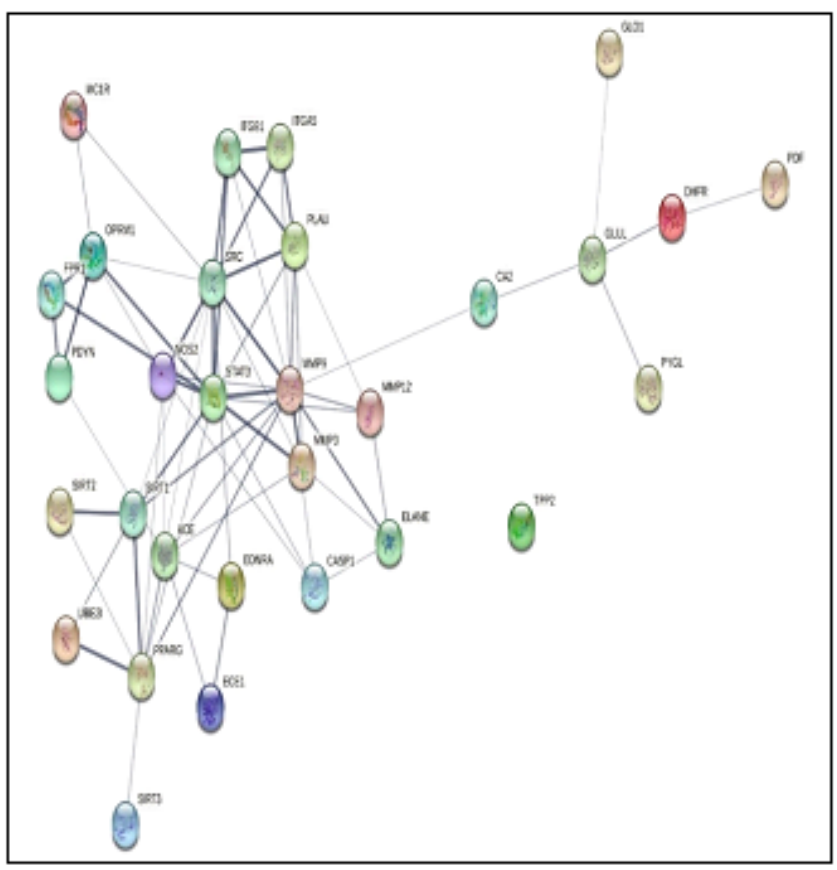

(B)

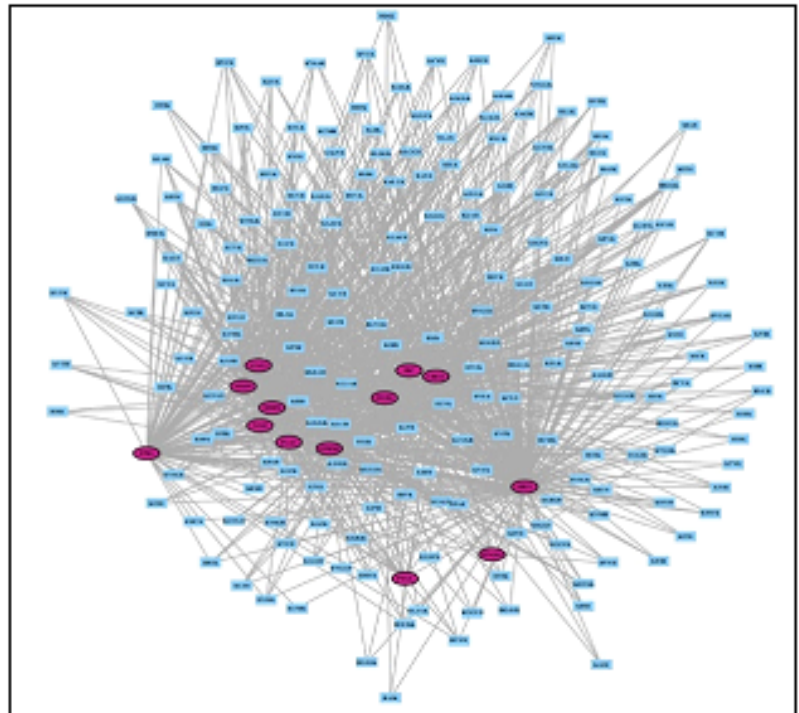

(D)

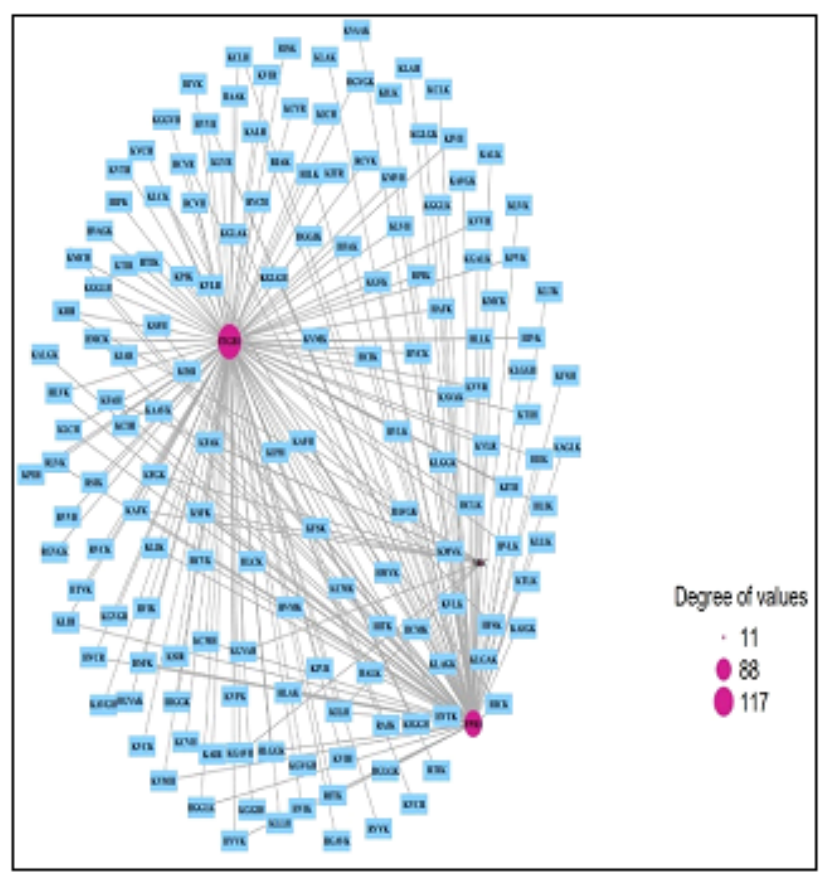

\section{Figure 3}

(A) The number of 11 signaling pathways on AMPs. (B) Networks of 11 signaling pathways on 210 nodes (197 Peptides, 13 Targets) and 1011 edges. (C) Protein-protein networks of 30 targets responded to bacterial infection (D) Size map of Rap1 signaling pathway on SRC, FRC1, and ITGB1 targets (158 nodes and 216 edges). 
(A)

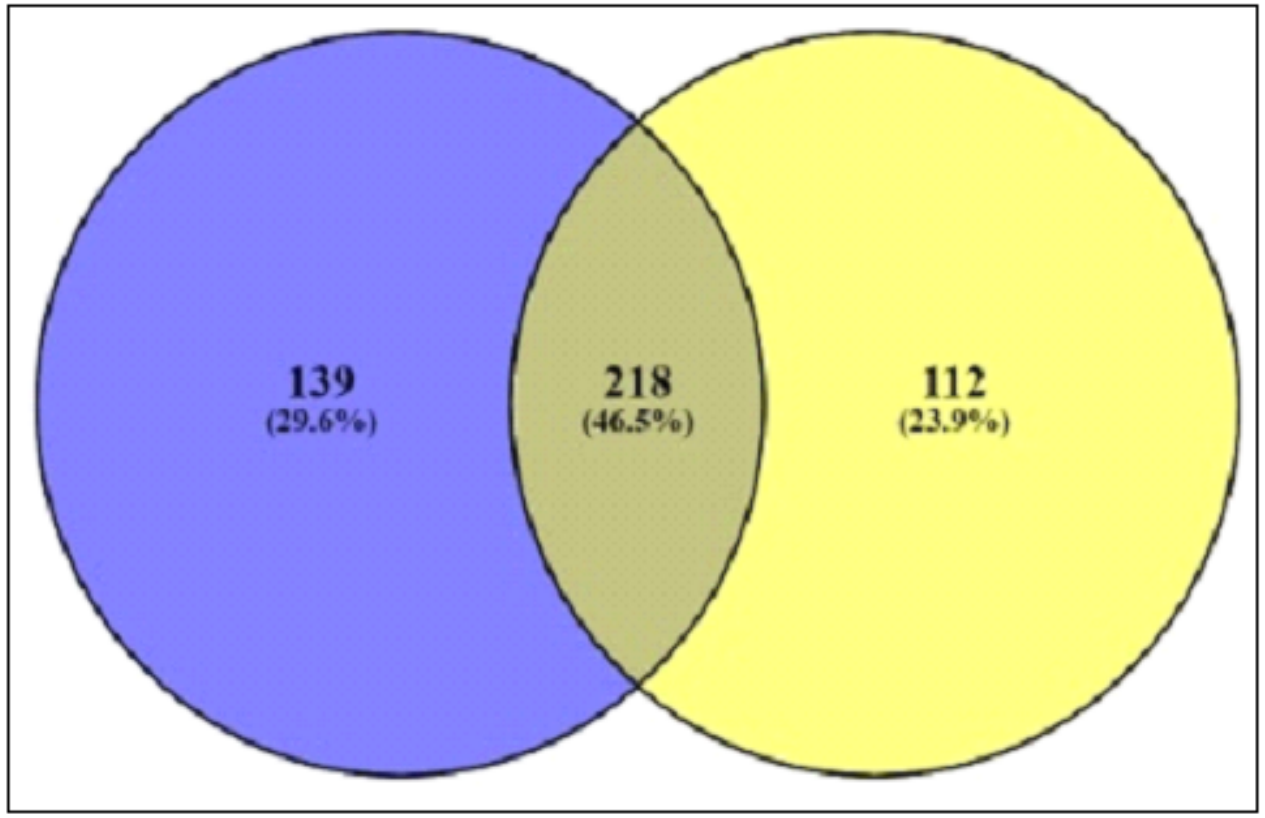

(B)

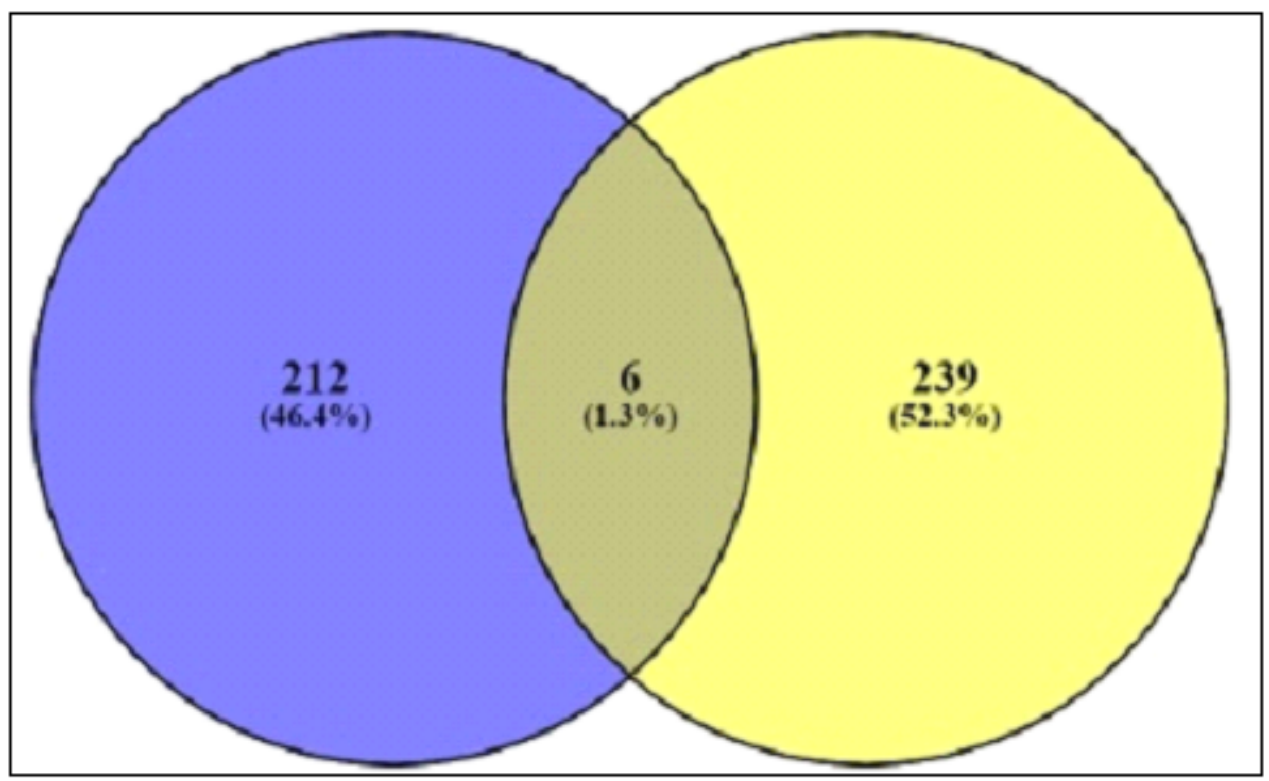

Figure 4

(A) The overlapping 218 targets identified by SEA (357 targets) and STP (330 targets) on AFPs-targets.

(B) The number of 6 overlapping targets between the number of 218 overlapping targets and 245 fungalrelated targets. 
(A)

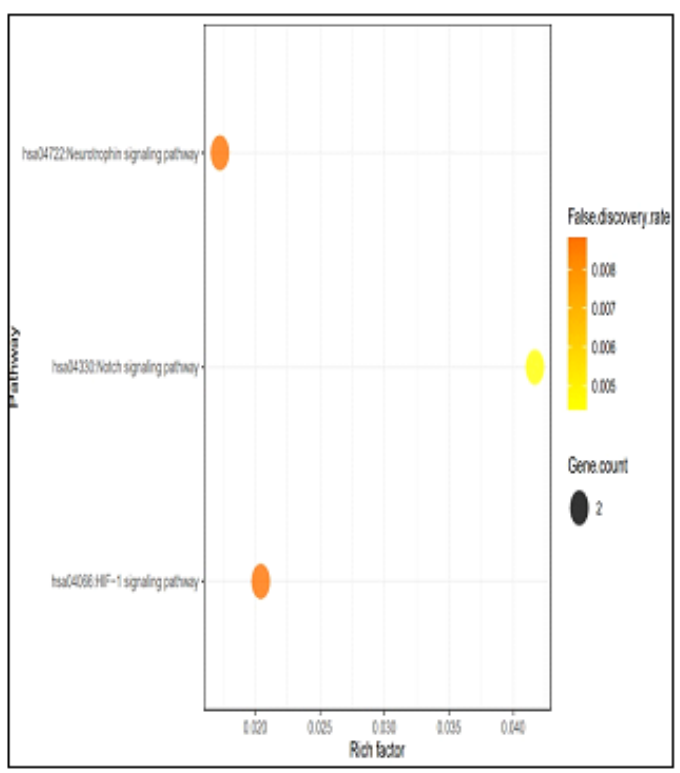

(C)

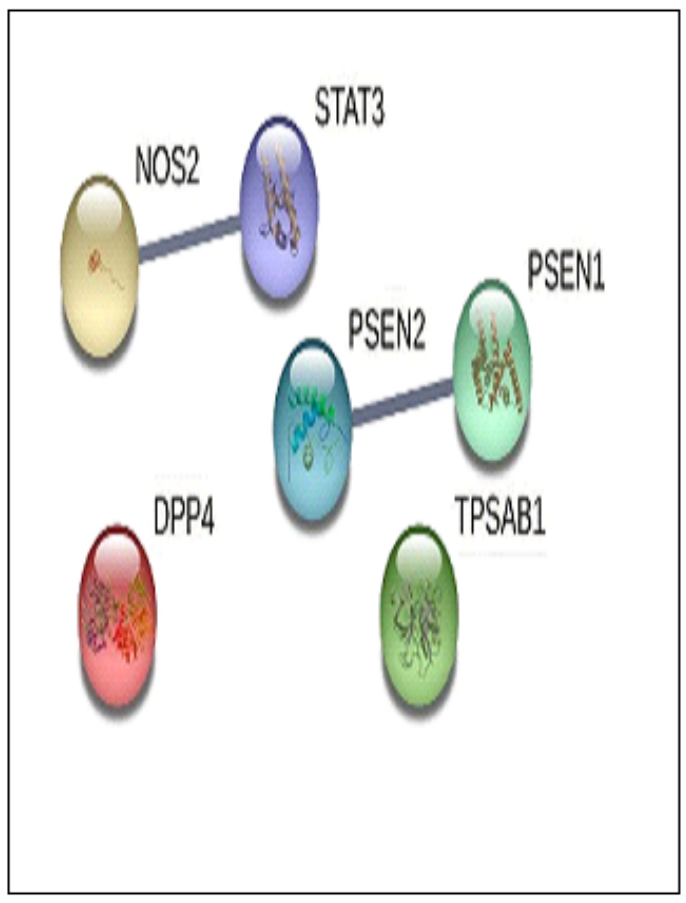

(B)

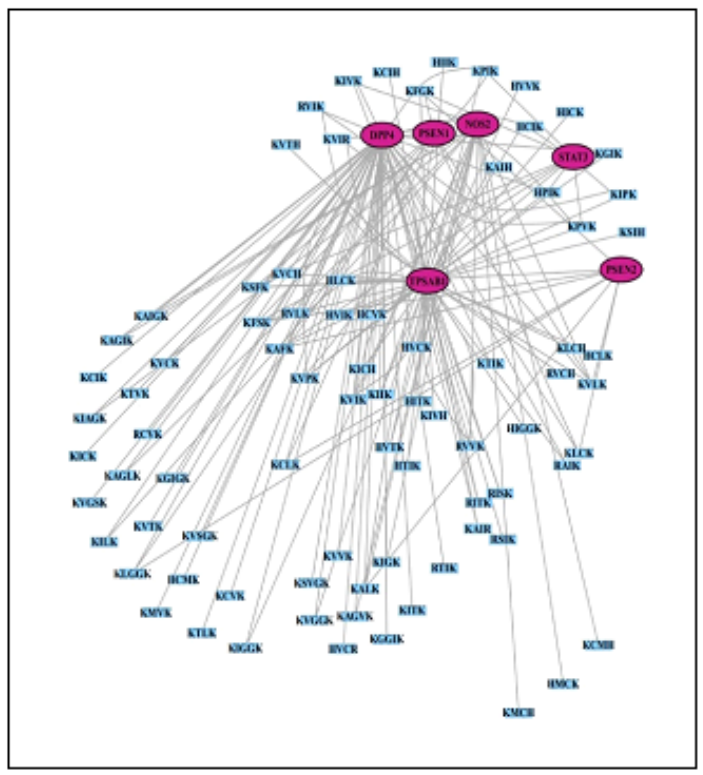

(D)

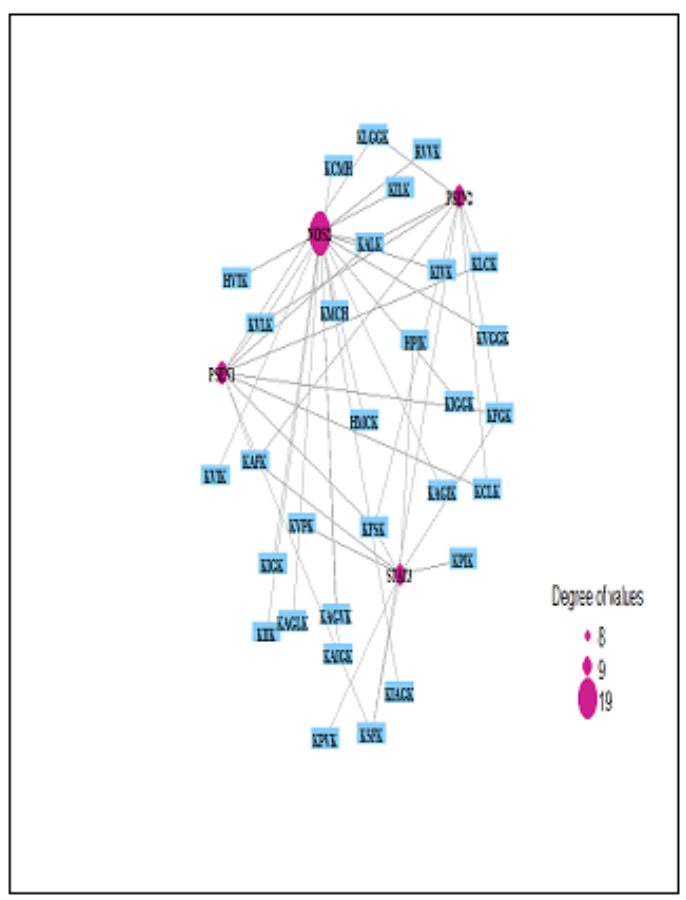

Figure 5

(A) The number of 3 signaling pathways on AMPs-AFPs axis (B) Networks of 3 signaling pathways on 87 nodes (81 peptides and 6 targets) and 150 edges. (C) Protein-protein networks of AMPs-AFPs (6 targets) (D) Size map of Notch signaling pathways on NOS2, STAT3, PSEN1, and PSEN2 (34 nodes and 45 edges). 
(A)

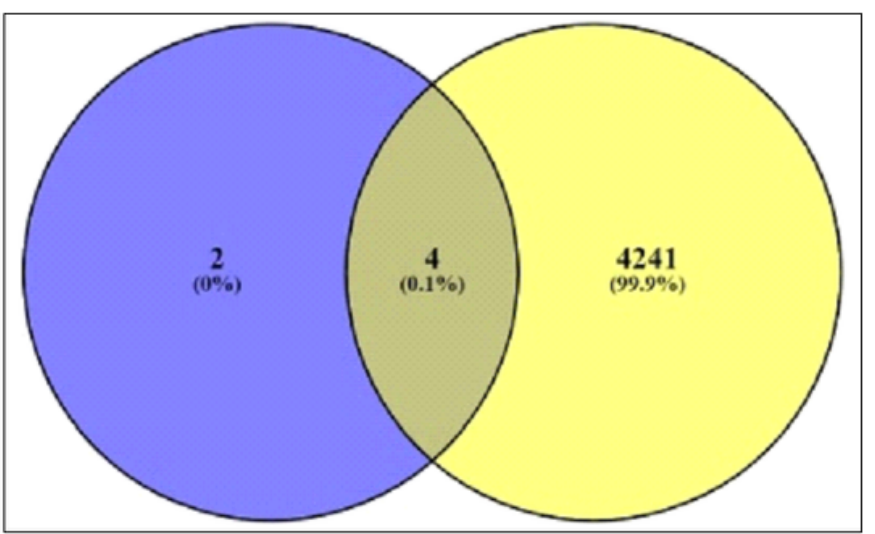

(B)

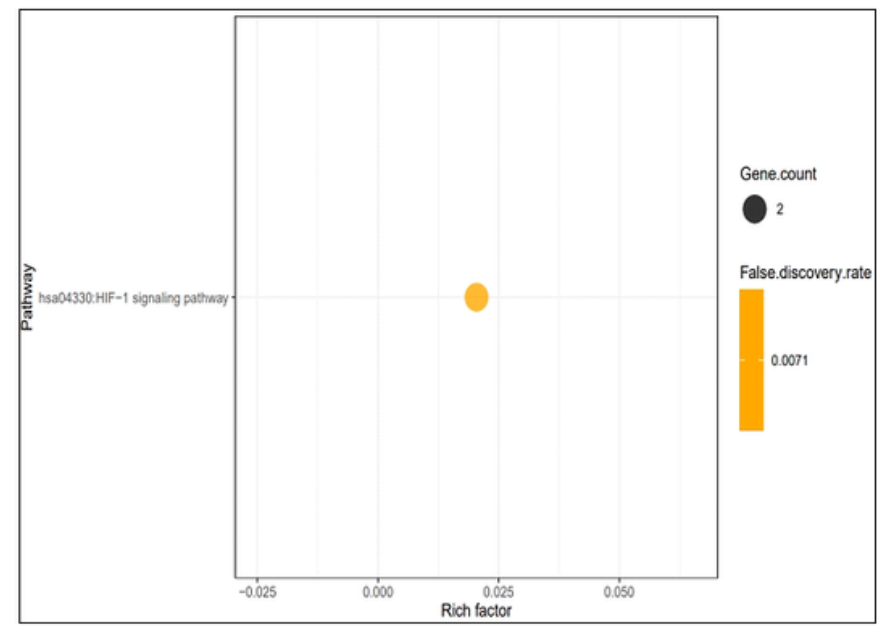

(C)

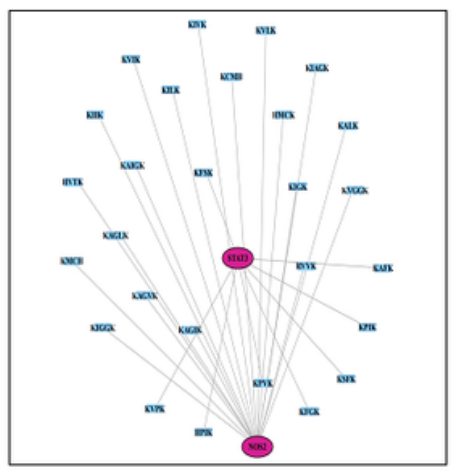

(D)

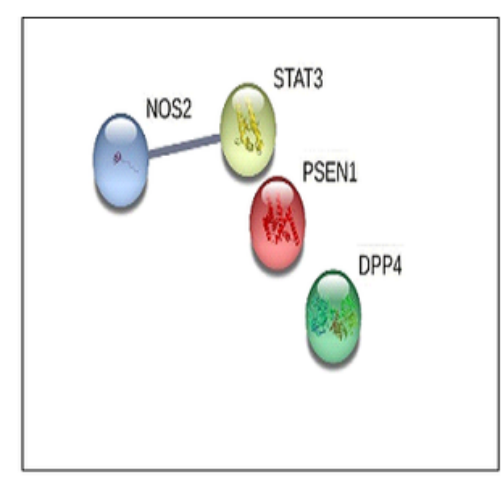

(E)

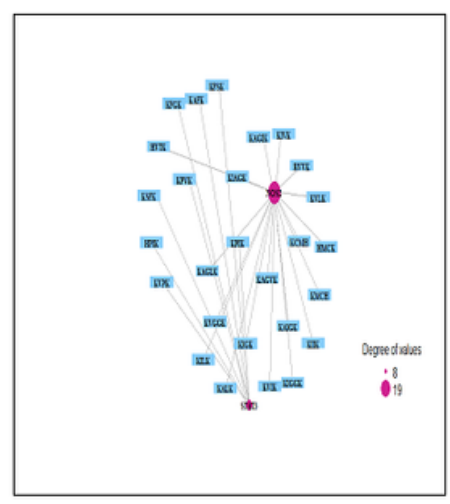

Figure 6

(A) Overlapping targets (4 targets) between AMPs-AFPs axis (6 targets) and cancer- related targets (4,245 targets). (B) The number of 1 signaling pathway on AMPs-AFPs-ACPs axis (C) Networks of HIF-1 signaling pathway on 29 nodes (27 peptides and 2 targets) and 27 edges. (D) Protein-protein networks of AMPs-AFPs-ACPs axis (4 targets) (E) Size map of HIF-1 signaling pathway on NOS2 and STAT3 (29 nodes and 27 edges). 
(A)

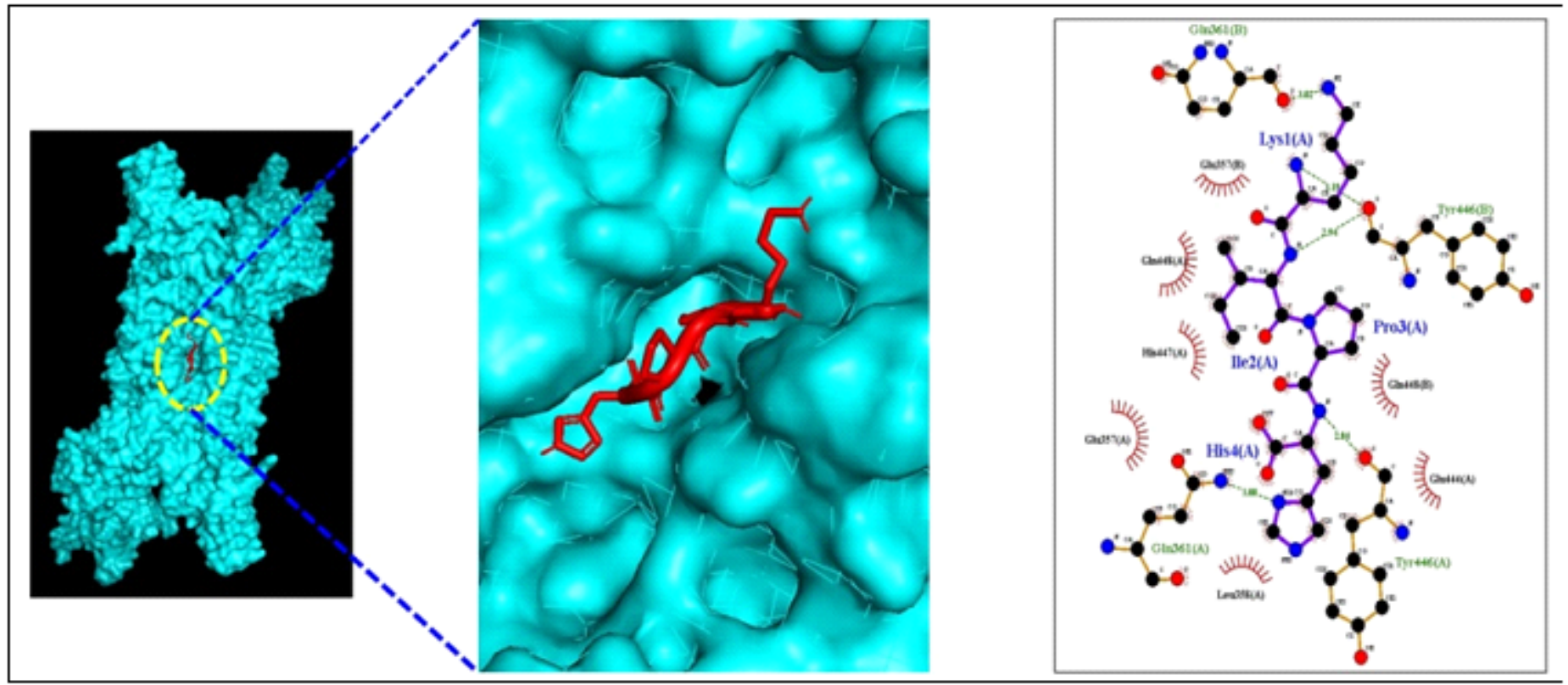

(B)

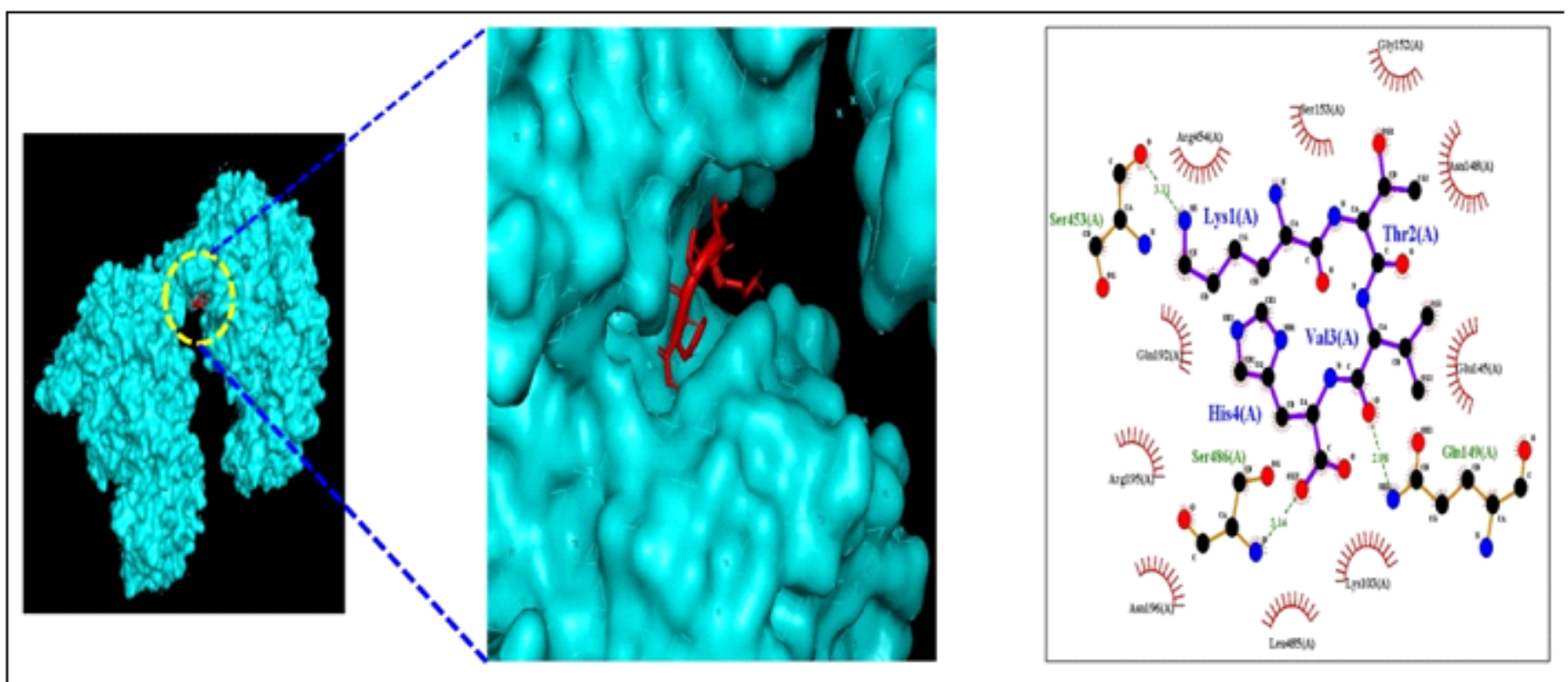

Figure 7

Molecular docking interaction between best docked SCPs and targets. (A) HPIK on STAT3 (PDB ID: 6TLC) (B) HVTK on NOS2 (PDB ID: 4NOS).

\section{Supplementary Files}

This is a list of supplementary files associated with this preprint. Click to download. 
- SupplementaryTableS1.pdf

- SupplementaryTableS10.pdf

- SupplementaryTableS11.pdf

- SupplementaryTableS12.pdf

- SupplementaryTableS2.pdf

- SupplementaryTables3.pdf

- SupplementaryTables4.pdf

- SupplementaryTableS5.pdf

- SupplementaryTableS6.pdf

- SupplementaryTables7.pdf

- SupplementaryTableS8.pdf

- SupplementaryTableS9.pdf 